SANDIA REPORT

SAND98-0701 - UC-405

Unlimited Release

Printed April 1998
SAND--98-ONO 121998

RECEIVED

MAY 271998

OSTI

\title{
A User's Guide for BREAKUP: A Computer Code for Parallelizing the Overset Grid Approach
}

Daniel W. Barnette

Prepared by

Sandia National Laboratories

Albuquerque, New Mexico 87185 and Livermore, California 94550

Sandia is a multiprogram laboratory operated by Sandia Corporation,

a Lockheed Martin Company, for the United States Department of

Energy under Contract DE-AC04-94AL85000.

Approved for public release; further dissemination unlimited:

\section{Sandia National Laboratories}
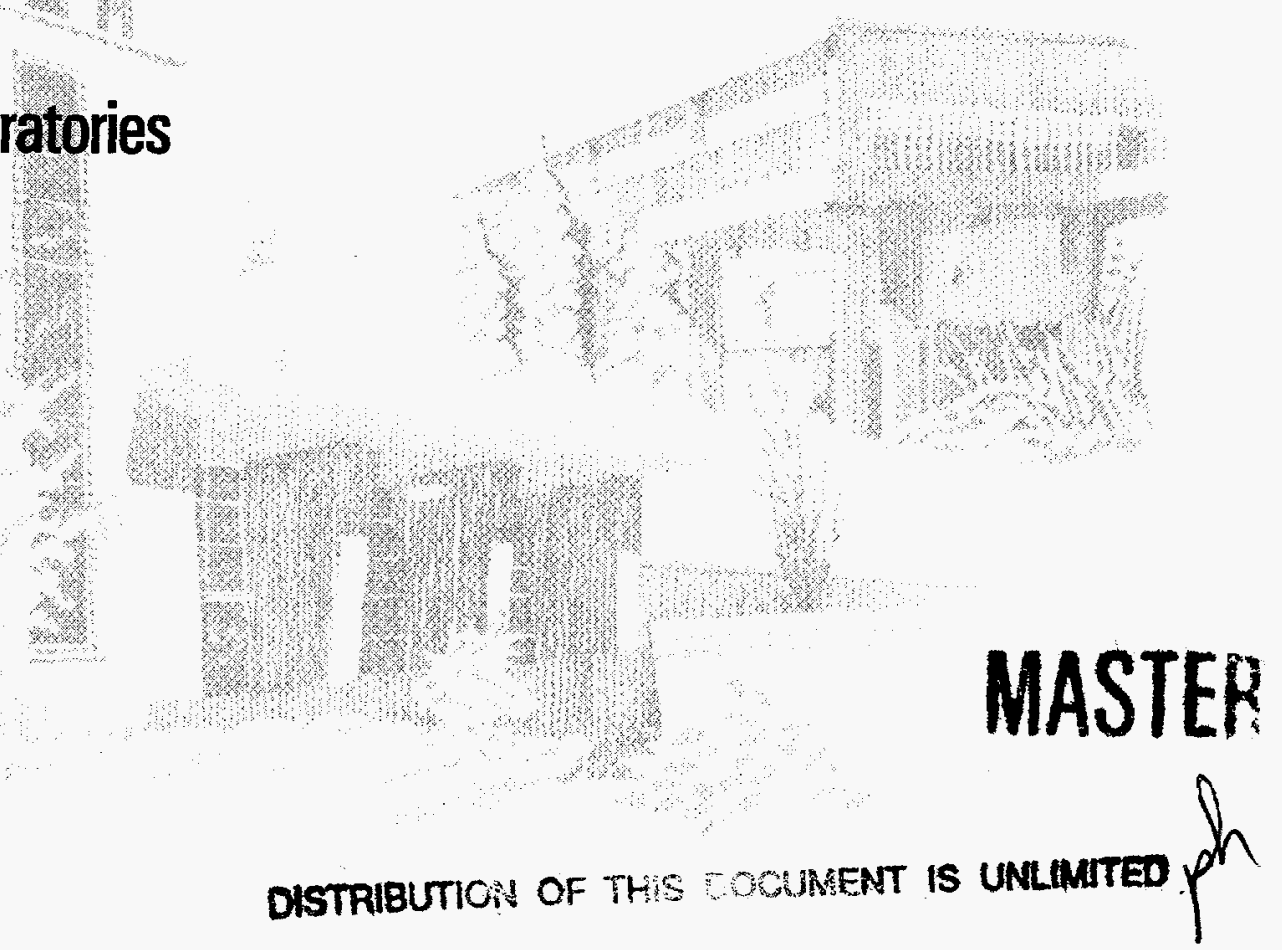
Issued by Sandia National Laboratories, operated for the United States Department of Energy by Sandia Corporation.

NOTICE: This report was prepared as an account of work sponsored by an agency of the United States Government. Neither the United States Government nor any agency thereof, nor any of their employees, nor any of their contractors, subcontractors, or their employees, makes any warranty, express or implied, or assumes any legal liability or responsibility for the accuracy, completeness, or usefulness of any information, apparatus, product, or process disclosed, or represents that its use would not infringe privately owned rights. Reference herein to any specific commercial product, process, or service by trade name, trademark, manufacturer, or otherwise, does not necessarily constitute or imply its endorsement, recommendation, or favoring by the United States Government, any agency thereof, or any of their contractors or subcontractors. The views and opinions expressed herein do not necessarily state or reflect those of the United States Government, any agency thereof, or any of their contractors.

Printed in the United States of America. This report has been reproduced directly from the best available copy.

Available to $\mathrm{DOE}$ and $\mathrm{DOE}$ contractors from

Office of Scientific and Technical Information

P.O. Box 62

Oak Ridge, TN 37831

Prices available from (615) 576-8401, FTS 626-8401

Available to the public from

National Technical Information Service

U.S. Department of Commerce

5285 Port Royal Rd

Springfield, VA 22161

NTIS price codes

Printed copy: A04

Microfiche copy: A01

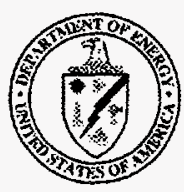




\section{DISCLAIMER}

Portions of this document may be illegible electronic image products. Images are produced from the best available original document. 


\title{
A User's Guide for BREAKUP: A Computer Code for Parallelizing the Overset Grid Approach
}

\author{
Daniel W. Barnette \\ Parallel Computational Sciences department \\ Sandia National Laboratories \\ Albuquerque, New Mexico 87185-1111 \\ Email: dwbarne@cs.sandia.gov
}

\begin{abstract}
In this user's guide, details for running BREAKUP are discussed. BREAKUP allows the widely used overset grid method to be run in a parallel computer environment to achieve faster run times for computational field simulations over complex geometries. The overset grid method permits complex geometries to be divided into separate components. Each component is then gridded independently. The grids are computationally rejoined in a solver via interpolation coefficients used for grid-to-grid communications of boundary data. Overset grids have been in widespread use for many years on serial computers, and several well-known Navier-Stokes flow solvers have been extensively developed and validated to support their use. One drawback of serial overset grid methods has been the extensive compute time required to update flow solutions one grid at a time. Parallelizing the overset grid method overcomes this limitation by updating each grid or subgrid simultaneously. BREAKUP prepares overset grids for parallel processing by subdividing each overset grid into statically load-balanced subgrids. Two-dimensional examples with sample solutions, and threedimensional examples, are presented.
\end{abstract}




\section{Acknowledgements}

The author wishes to acknowledge Dr. Curtis C. Ober of Sandia's Parallel Computational Science Department for his help in modifying a two-dimensional incompressible NavierStokes code to run on the Intel Paragon with output from BREAKUP. 


\section{Contents}

Introduction

The Parallel Overset Grid Approach

a) Overview

b) Overset Grid-to-Grid Communications

c) Parallel Overset Grid Construction $\quad 7$

How BREAKUP Prepares Grids for Parallel Processing

a) Load balance $\quad 8$

b) Speed-up 9

c) Sample BREAKUP Output 9

d) Construction of Connectivity Tables 13

$\begin{array}{ll}\text { Running BREAKUP } & 14\end{array}$

User Options

a) Option 1 - PEGSUS-formatted Generic Overset Grids 14

b) Option 2 - PLOT3D-formatted Multiple Grids, No Interpolation Coefficients, Subgrid Overlap Required $\quad 15$

c) Option 3 - PLOT3D-formatted Multiple Grids, No Interpolation Coefficients, No Subgrid Overlap Required; or Internally Generated Grid 15

Input Files 16

$\begin{array}{ll}\text { Output Files } & 17\end{array}$

Examples $\quad 17$

$\begin{array}{ll}\text { Future Work and Directions } & 18\end{array}$

$\begin{array}{ll}\text { Summary } & 19\end{array}$

References $\quad 20$

Appendices
A: BREAKUP Subroutines and Their Description

B: List of Variables used in BREAKUP $\quad 51$

C: Sample Output from BREAKUP (file BREAKUP.OUT)

Figures

1. Overview flow chart for the parallel overset grid method 21

2. Overview flow chart for BREAKUP when overset grid output ... 22

3. Illustration of increase in grid-to-grid and intra-grid communications... 23

4. BREAKUP's subroutines listed in alphabetical order 24

5. BREAKUP calling tree 25

6. Cube illustrating how BREAKUP generates 74 subgrids 26

7. BREAKUP-generated subgrids for a multi-element airfoil grid... 27

8. BREAKUP subgrids for an F16's forward fuselage and intake duct 29

9. Original six overset grids for the Gulf of Mexico, with serial solution 30

10. Sixteen BREAKUP-generated overset grids for the Gulf of Mexico 34

11. One hundred BREAKUP-generated overset subgrids for the Gulf of Mexico 36 


\section{A User's Guide \\ for \\ BREAKUP: A Computer Code \\ for Parallelizing the Overset Grid Approach}

\section{Introduction}

The overset grid approach for computing field simulations around geometrically complex configurations has become widely used since its inception in the early 1970's[1]. The attractiveness of the approach lies in the fact that a complex geometry can be divided into its inherently simpler parts while maintaining geometric complexity. Each part is then gridded separately. This is presumed to be much less complicated a process than suitably gridding the entire geometry alone. The grids are computationally re-combined in a solver in such a manner as to give a smooth solution within and across each grid domain. The approach has continued to mature in the serial computing environment. The wide acceptance of the method is reflected in the fact that overset grids have been implemented in well-known compressible as well as incompressible Navier-Stokes flow solvers.

Until the paradigm of parallel computing was developed, one of the major drawbacks to the overset grid approach was the necessity of updating the solutions on each grid separately. This involves sequentially reading each grid into core, updating the flow field one time step, and re-writing the updated solution back to disk storage. The next grid is then read into core and the process repeated. Each grid receives its updated boundary conditions from other grids via interpolation coefficients. The coefficients are computed using other codes after the overset grids are generated but before the grids are submitted to a flow solver. Since iterative solvers typically require on the order of thousands of time steps for convergence, wall-clock time can be excessive when grids are swapping in and out of core. Additionally, time spent in queues during run time can result in significant delays for solutions that would require orders of magnitude less wall clock time had the user sole use of the computer.

The drawbacks noted above may be overcome by a parallel approach to the overset grid method. The 'parallelized overset grid approach' discussed herein involves dividing each of the several main grids into an arbitrary number of load-balanced subgrids, with each subgrid assigned to a processor in a distributed or massively parallel computing environment. The previously generated interpolation coefficients for grid-to-grid communications are analyzed and reassigned (not regenerated!) to their corresponding subgrid processor so that each knows which processor will need its updated dependent variables and which processors will be sending updated dependent variables to it. The solvers must be modified to run on parallel computers so that each subgrid can now be updated simultaneously rather than sequentially. This approach leads to significantly faster run times for more complex problems than could have been modeled in the past. 
This user's guide discusses the computer code BREAKUP. The BREAKUP code was developed to subdivide overset grids in a manner that load balances the application on parallel computers. The code provides the capability to generate parallel solutions using overset grids in solvers modified for parallel computers. The code has been written so that a minimum of changes will be needed to modify a solver for parallel applications.

\section{The Parallel Overset Grid Approach}

\section{a) Overview}

An overview flow chart for the parallel overset grid method is presented in Fig. 1. The flow chart illustrates the point that the method was designed to minimally impact the serial overset grid approach. The serial-to-parallel modifications needed to implement the approach involve the requirement that the grids need additional preparation for the parallel solver, and that the solver must be modified via message passing calls for use on a parallel computer. The method currently implemented is such that all codes, i.e. BREAKUP, visualization codes, and pre- and post-processing codes, run on a workstation. The solver, of course, is run on a parallel computer.

\section{b) Overset Grid-to-Grid Communications}

Overset grids require the capability to communicate with overlapped or embedded grids. Currently, PEGSUS 4.0[2] is used to generate interpolation coefficients for grid-to-grid communications where separate grids overlap. Solvers that are PEGSUS-based use the interpolation coefficients and data from other influencing grids to update boundary conditions in overlapped regions on each grid as the governing equations are solved. Users should consult Ref. 2 for more detailed information.

Other codes exist to generate the interpolation coefficients. However, as currently written, BREAKUP is configured to read the output of PEGSUS only.

\section{c) Parallel Overset Grid Construction}

BREAKUP may be considered as a preprocessor to PEGSUS-based parallel solvers and, as such, represents the cornerstone to the parallel overset grid approach. An overview flow chart of BREAKUP is presented in Fig. 2. The primary purpose of BREAKUP is to prepare the output of PEGSUS for parallel computing. PEGSUS output includes the overset (known as 'composite' in PEGSUS jargon) grids, the 'iblank' array that flags grid points for special handling in the solver, and an interpolation coefficient file used for grid-to-grid communication. BREAKUP divides each overset grid into subgrids corresponding to the user-specified number of processors on a parallel computer or in a distributed computing environment. The code computes the total number of grid points, computes the average number of grid points per processor, and divides each grid into subgrids such that a near-uniform distribution of grid points exists on each processor. Separate overset grids are divided such that the grid's perimeter-to-area ratio in $2-\mathrm{D}$, or surface-to-volume ratio in $3-\mathrm{D}$, is minimized. The subgrids are assigned to individual 
processors in a sequential manner with no regard to optimal communication paths between adjacent processors. This means that there is no guarantee that two subgrids from different overset grids requiring high communications costs will lie adjacent or even close to each other to minimize latency. This is an area for further algorithmic development.

Dividing the overset grids into subgrids results in a significant amount of message passing between processors, as illustrated in Fig. 3. This is due to the necessity for gridto-grid communications as well as intra-grid communication. Intra-grid communication is defined as message passing between subgrids of a unique overset grid. Grid-to-grid communication is defined as message passing between subgrids of separate overset grids. For grid-to-grid communications, the additional boundaries resulting from forming subgrids requires appropriate interpolation coefficients corresponding to each new subgrid. The interpolation coefficients are the same as those computed by the PEGSUS code. No new data are generated in BREAKUP. BREAKUP searches these coefficients and correlates them to the appropriate subgrids.

As mentioned above, intra-grid communication results from overlapping subgrids of an individual, unique overset grid. Adjacent subgrids align point-to-point by definition and, therefore, require no interpolation coefficients per se. However, the solver must know where to send and receive boundary information for intra-grid, as well as grid-to-grid, communications. BREAKUP constructs connectivity tables for both grid-to-grid and intra-grid communications such that each processor has the correct data to update its subgrid. The table for grid-to-grid communications contains the processor location of each pair of interpolation coefficients and those points on other processors (i.e., subgrids) which require the coefficients. The solver must be modified to use the tables to update grid boundary data. More detail regarding connectivity tables is given below.

\section{How BREAKUP Prepares Grids for Parallel Processing}

Two primary issues in parallel processing are load balance and speed-up. These issues, and how they are dealt with in BREAKUP, will now be considered. Also discussed is the construction of connectivity tables used in the user-modified solver to define processorto-processor communications.

\section{a) Load balance}

Load balancing on parallel processors means that each processor should have the same, or nearly the same, work. In practice, this can be difficult to precisely achieve. The inherent power of the overset grid method lies in the fact that the size of individual overset grids can significantly vary in any given problem.

The approach to load-balancing subgrids in BREAKUP is as follows. First, BREAKUP determines the total number of grid points in all grids and divides by the number of userspecified subgrids (or processors) required. This gives the average number of grid points 
per processor. Next, the number of grid points in each individual overset grid is divided by this number to give the total number of processors that will be dedicated to that grid. Of course, this number seldom, if ever, is a whole number. BREAKUP is programmed to sequentially enlarge subgrid boundaries within the said grid to encompass more grid points that remain after calculating the number of processors per grid. This process ensures that the subgrid boundaries remain flexible enough to encompass all grid points. Also, it keeps the impact on load balancing to a minimum while keeping fixed the number of processors assigned to that grid.

If it happens that many grid points are left over, it becomes possible to better load balance the original grids with a larger number of processors. In this case, the code outputs a message to the effect that the grids cannot be optimally subdivided on the specified number of processors. BREAKUP then calculates the nearest number of processors over which the grids would be better load balanced. The user is then queried to either accept the new number and continue or input a new value for the number of processors required.

It is possible that BREAKUP will be given a small enough grid that cannot be subdivided. In this case, BREAKUP will leave the small grid intact. The premise is that, if there are only a few, these relatively small grids will not have a significant impact on the load balancing of the entire set of overset grids. That is, only a few processors with very small grids will have to wait on the many other processors to finish a solution time step.

\section{b) Speed-up}

Speed-up occurs by minimizing communications between subgrids and maximizing the computational work performed on each processor. Communication occurs at the grid boundaries and hence is proportional to the grid's surface area. Computational work is performed at each grid point and is therefore proportional to the grid volume. Hence, it is advantageous when subdividing the grid to minimize its surface area and maximize its volume. Once the number of processors assigned to each overset grid is known, BREAKUP determines all the 3-factors of that number and finds the minimum value of the ratio of subgrid surface area to volume.

\section{c) Sample BREAKUP output}

An example of how BREAKUP handles load-balancing and speed-up will illustrate the process.

Assume a user has the following six grids, with the grid size indicated by $j, k$, and $l$. Each grid has the total number of points listed. 


$\begin{array}{ccccc}\text { Grid\# } & j & k & l & \text { Total Points } \\ -1 & 3 & 77 & 53 & 12243 \\ 2 & 3 & 249 & 20 & 14940 \\ 3 & 3 & 240 & 20 & 14400 \\ 4 & 3 & 135 & 20 & 8100 \\ 5 & 3 & 234 & 20 & 14040 \\ 6 & 3 & 133 & 20 & 7980\end{array}$

The total number of grid points is 71,703 . If the user chooses to subdivide these grids into 16 subgrids, the average number of points per processor (rounded to the nearest integer) is 4,481 . Dividing the number of grid points for each grid by the average number of gridpoints desired on each processor yields the following information:

\begin{tabular}{cccc} 
Grid \# & $\begin{array}{c}\text { Avg. no of grid } \\
\text { points per } \\
\text { processor }\end{array}$ & $\begin{array}{c}\text { No. of } \\
\text { processors } \\
\text { for each grid }\end{array}$ \\
\hline 1 & 12243 & 4481 & 3 \\
2 & 14940 & 4481 & 3 \\
3 & 14400 & 4481 & 3 \\
4 & 8100 & 4481 & 2 \\
5 & 14040 & 4481 & 3 \\
6 & 7980 & 4481 & 2
\end{tabular}

BREAKUP calculates that three processors will be assigned to Grid \#1, three to Grid \#2, and so on. This implies, of course, that Grid \#1 will be divided into three subgrids, Grid \#2 into three subgrids, etc. Although this simple calculation informs the user how many subgrids will be formed from each original overset grid, it does not indicate how the subgrids will be formed. To do this, BREAKUP examines all of the three-factors that can be determined from the number of processors assigned for each grid. For Grid \#1, BREAKUP determines that the original grid can be subdivided in the following ways.

$\begin{array}{cccc}\text { ITAG } & \text { J } & \text { K } & \text { L } \\ --- & -- & --- & - \\ 1 & 1 & 1 & 3 \\ 2 & 1 & 3 & 1 \\ 3 & 3 & 1 & 1\end{array}$

The ITAG parameter is for reference only. The row-wise product of the three-factors listed under the $J, K$, and $L$ columns is always equal to the number of processors assigned to Grid $\# 1$. Here, $J, K$, and $L$ are assumed to be oriented in the grid's $j, k$, and $l$ direction, respectively, and represent the maximum indices that will be assigned to the subgrids. For example, Grid \#1 can be subdivided into 1 subgrid in the grid's own $j$ direction, 1 subgrid in its $k$ direction, and 3 subgrids in its $l$ direction. Any permutation of this can also be made. However, the permutation desired is the one that will yield a minimum surface area for each subgrid and a maximum grid volume. Therefore, each combination 
of three-factors, associated with increasing values of ITAG, must be examined to give the minimum ratio of subgrid area over subgrid volume.

The surface area of any one subgrid for Grid \#1 may be written as

$$
\text { surf }=2\left[\left(\frac{j \max }{J} \times \frac{k \max }{K}\right)+\left(\frac{k \max }{K} \times \frac{l \max }{L}\right)+\left(\frac{j \max }{J} \times \frac{l \max }{L}\right)\right]
$$

where jmax, kmax, and lmax are the dimensions of the original overset grid, and $J, K$, and $L$ are the number of subgrids into which the grid will be subdivided in their respective directions. Hence, the quantity $j m a x / J$ will denote the dimension, or number of grid points, of one subgrid in the $j$ direction. Note that $J, K$, and $L$ can take on any permutation of the three-factors listed above. The volume of any of the subgrids is given by

$$
v o l=\frac{j \max }{J} \times \frac{k \max }{K} \times \frac{l \max }{L}
$$

The area-to-volume ratio is given by

$$
\frac{\text { surf }}{\text { vol }}=2\left(\frac{J}{j \max }+\frac{K}{k \max }+\frac{L}{l \max }\right)
$$

This ratio is to be minimized to achieve the most efficient configuration for subdividing the original grid. Minimization occurs by substituting the various three-factors for $J, K$, and $L$ associated with the variable ITAG. The ratio is calculated for each permutation. The combination that gives the minimum ratio is selected as the way to subdivide the grid. For Grid \#1, BREAKUP calculates the following ratios for each ITAG three-factors.

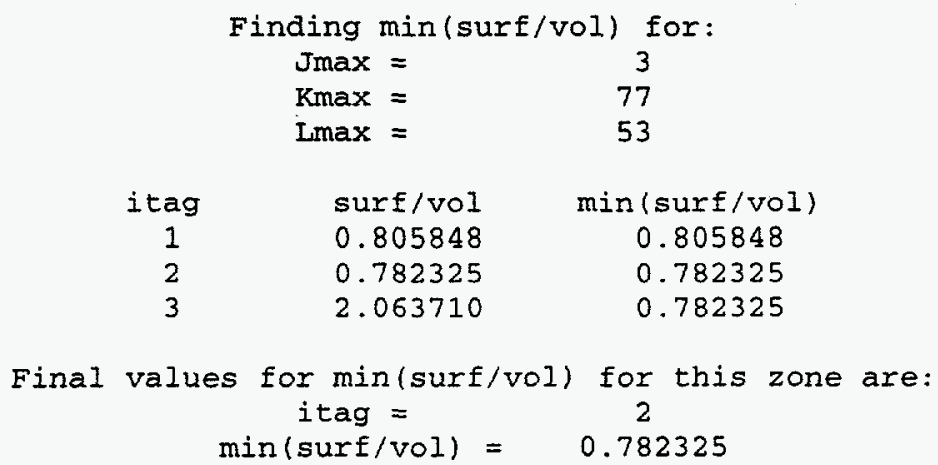

Once the particular three-factor is determined that yields the minimum surface-to-volume ratio, then the grid points in the directions corresponding to the appropriate ITAG value chosen above are divided by the appropriate $J, K$, or $L$ value to give the approximate 
number of points per subgrid in each direction after selecting the ITAG=2 permutation. BREAKUP's output for this is as follows.

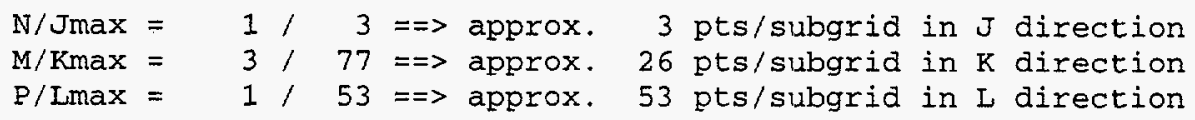

where $N, M$, and $P$ are used to denote the permuted $J, K$, and $L$ values for $I T A G=2$. Note that 26 points per subgrid in the $K$ direction for three subgrids will leave one point too many in that direction. BREAKUP handles this as indicated by the following output.

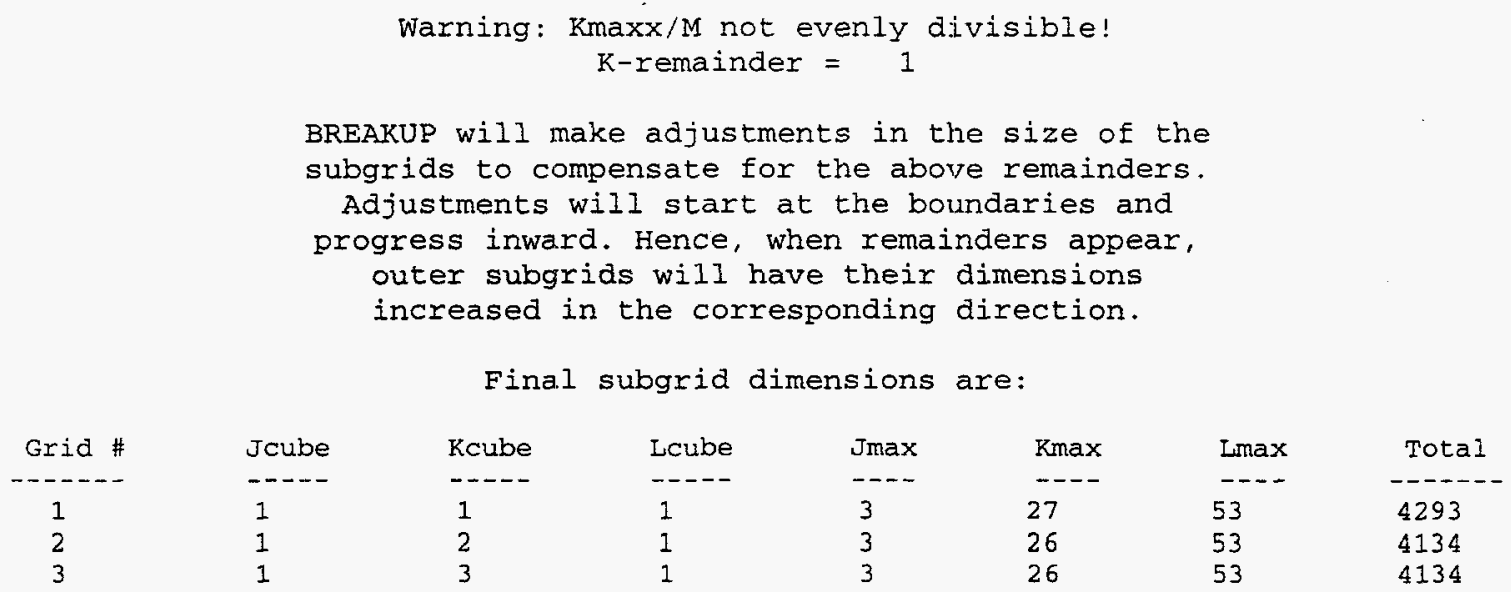

The values for Jcube, Kcube, and Lcube denote the indices given to each subgrid. The values listed for Jmax, Kmax, and Lmax denote the number of points in the corresponding subgrid along each of its sides. Finally, the total number of grid points for each subgrid in Grid \#1 is listed in the Total column. The totals include grid points on common and overlapped faces between subgrids. It can be seen the values are within approximately $8 \%$ of the 'average no. of grid points per processor' value of 4,481 listed above.

To ensure accuracy, BREAKUP calculates the total number of grid points in all of the subgrids, subtracts the grid points in the common or overlapped regions that were formed as indicated above, and compares the final number with the total number of grid points originally computed for the overset grid. The user is notified if any discrepancies exist. In the present example, the number computed from subgrids and the original number match, as they should, and as indicated in the following output.

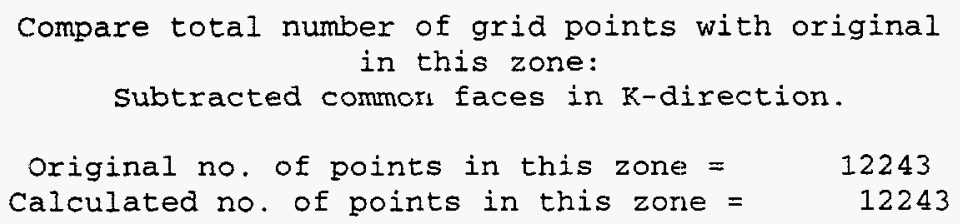


The above process is repeated for each overset grid. If the user has input PEGSUSgenerated interpolation coefficients, BREAKUP will correlate the original coefficients with the new subgrids for constructing the connectivity tables, discussed below.

Note that the best speed-up in the above sense would be to have the maximum number of grid points in the maximum amount of memory allowed on processors having identical amounts of memory. This may be a realistic expectation for homogeneous compute clusters or massively parallel machines. It is not realistic for heterogeneous compute clusters. Due to possibly widely varying cpu's, cache, memory, bandwidth, etc., heterogeneous clusters present a significant challenge to any code attempting to achieve a high degree of parallelism. BREAKUP currently does not account for variance of any hardware limitations when subdividing grids.

\section{d) Construction of Connectivity Tables}

Connectivity tables are needed by the solver to determine which processors need to send information to which receiving processors and vice versa. These tables contain information to be used for passing information from processor to processor. Shown below are partial sample outputs for connectivity tables as generated by BREAKUP. Two tables are constructed. One is generated for intra-grid communications; the other, for grid-to-grid communications. The table for intra-grid communications will be discussed first.

A sample connectivity table for intra-grid communications is listed below. The first column indicates the appropriate subgrid under attention. The remaining part of the row lists the subgrid range of indices from which messages will be sent. The next row indicates the subgrid and subgrid indices that will receive information from the processor indicated in the line immediately above it. Hence, each pair of rows indicate a subgrid and its boundaries from which data will be sent, and which subgrid and boundary will receive the data. For example, subgrid 1 will communicate with subgrid 2 , as indicated in the first two rows of numbers. The next two rows indicate subgrid 2 will communicate with subgrid 3 . Next, it is indicated that subgrid 2 will also communicate with subgrid 1 , 3 with 2,4 with 5 , and so on.

$\begin{array}{crrrrrrrrr}\text { cone\# } & \text { J_beg } & J_{-} \text {end } & J_{-} \text {inc } & \text { K_beg } & \text { K_end } & \text { K_inc } & \text { L_beg } & \text { L_end } & \text { L_inc } \\ 1 & 1 & 3 & 1 & 27 & 27 & 1 & 1 & 53 & 1 \\ 2 & 1 & 3 & 1 & 2 & 2 & 1 & 1 & 53 & 1 \\ 2 & 1 & 3 & 1 & 28 & 28 & 1 & 1 & 53 & 1 \\ 3 & 1 & 3 & 1 & 2 & 2 & 1 & 1 & 53 & 1 \\ 2 & 1 & 3 & 1 & 1 & 1 & 1 & 1 & 53 & 1 \\ 1 & 1 & 3 & 1 & 26 & 26 & 1 & 1 & 53 & 1 \\ 3 & 1 & 3 & 1 & 1 & 1 & 1 & 1 & 53 & 1 \\ 2 & 1 & 3 & 1 & 27 & 27 & 1 & 1 & 53 & 1 \\ 4 & 1 & 3 & 1 & 85 & 85 & 1 & 1 & 20 & 1 \\ 5 & 1 & 3 & 1 & 2 & 2 & 1 & 1 & 20 & 1\end{array}$

A sample connectivity table for grid-to-grid communications is presented below. The first line indicates the number of points (477) in subgrid \#1 whose data need to be sent to 
other subgrids. The subsequent columns list the three indices $(1,22,43)$ of the grid point in the donor subgrid $\# 1$, the three interpolation coefficients needed to compute the data needed, the processor number (\#4) to which data will be sent, and the three indices $(1,76,20)$ of the point in the recipient subgrid at which the dependent variables will be interpolated. The first few lines for donor subgrid \#2 are shown for continuity. The solver uses these tables to send and receive messages for updating grid boundaries.

\begin{tabular}{|c|c|c|c|c|c|c|c|c|c|c|}
\hline \multirow[t]{5}{*}{477} & Base & \multicolumn{2}{|c|}{ subzone } & \# 1 & & & & \multirow[b]{2}{*}{1} & \multirow[b]{2}{*}{76} & \multirow[b]{2}{*}{2} \\
\hline & 1 & 22 & 43 & $0.0000000 E+00$ & $6.2530622 \mathrm{E}-02$ & $3.8906133 \mathrm{E}-01$ & 4 & & & \\
\hline & 1 & 22 & 43 & $0.0000000 \mathrm{E}+00$ & $2.5979275 E-01$ & $4.271 .3684 E-01$ & 4 & 1 & 73 & 20 \\
\hline & 1 & 21 & 43 & $1.0000000 \mathrm{E}+00$ & $8.2356793 \mathrm{E}-01$ & $2.7348068 E-01$ & 4 & 2 & 79 & 20 \\
\hline & 2 & 21 & 43 & $1.0000000 E+00$ & $9.1715431 \mathrm{E}-01$ & $3.2546192 \mathrm{E}-01$ & 4 & 3 & 78 & 20 \\
\hline & & & & & - & & & & & \\
\hline & & & & & - & & & & & \\
\hline & \multirow{2}{*}{\multicolumn{3}{|c|}{ Base subzone }} & & • & & & & & \\
\hline \multirow{5}{*}{1035} & & & & $\#$ & & & & & & \\
\hline & 1 & 15 & 46 & $0.0000000 E+00$ & $2.9964754 \mathrm{E}-01$ & $9.7948408 E-01$ & 4 & 1 & 12 & 20 \\
\hline & 1 & 6 & 35 & $1.0000000 E+00$ & $2.9722536 \mathrm{E}-01$ & $6.7863387 \pm-01$ & 4 & 2 & 28 & 20 \\
\hline & 1 & 15 & 48 & $0.0000000 E+00$ & $5.0462323 E-01$ & $9.611 .3777 \mathrm{E}-01$ & 4 & 1 & 5 & 20 \\
\hline & 2 & 16 & 49 & $1.0000000 E+00$ & $5.2282799 \mathrm{E}-02$ & $3.2107067 \mathrm{E}-01$ & 4 & 3 & 2 & 20 \\
\hline
\end{tabular}

\section{Running BREAKUP}

BREAKUP has been modularly constructed to perform the task of preparing multiple overset grids for parallel processing. This allows the user to more easily run and, if necessary, modify the code. BREAKUP's subroutines are listed in alphabetical order in Fig. 4. A calling tree is presented in Fig. 5 for the reader's reference.

A brief description of each subroutine is listed in Appendix A. The purpose of each subroutine is listed, as well as the calling and called subroutines.

A variable list is presented in Appendix B. A brief description of the more significant: variables is given.

A complete BREAKUP output listing for a sample run in presented in Appendix C. Part of the sample output listings noted above were taken from this appendix. The grids used in the appendix correlate to Fig. 10, to be discussed.

When run, BREAKUP presents the user with three options for producing subgrids. The listings shown below duplicate the output from the code upon startup. The options should be self-explanatory. Note that the only significant input required from the user is the number of processors over which the original multiple overset grids must be subdivided.

\section{User Options}

\section{a) Option 1 - PEGSUS-formatted Generic Overset Grids}

This is the most powerful option. Output from the PEGSUS code, which includes the grids, interpolation coefficients, and other necessary data, is read in. The grids are then 
subdivided, connectivity tables constructed, and the data output for parallel processing. It also allows leaving the original grids intact but constructing connectivity tables for parallel processing. This implies that each overset grid would reside on one processor with no intra-grid message passing needed.

\section{1) BREAKUP OVERSET GRIDS WITH INTERPOLATION COEFFICIENTS FROM PEGSUS} OUTPUT

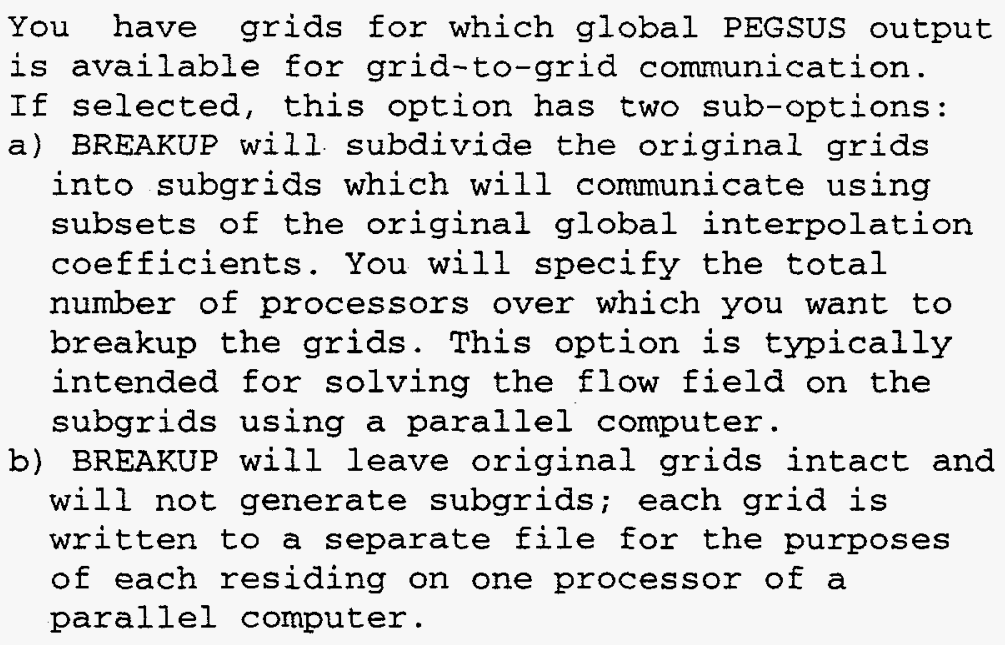

\section{b) Option 2 - PLOT3D-formatted Multiple Grids, No Interpolation Coefficients, Subgrid Overlap Enforced}

This is a versatile option. It was envisioned that an option was needed so that the user could prepare grids for parallel processing without necessarily having PEGSUS output. Hence, the user could examine the output of BREAKUP, for example, to aid in determining how the code subdivides the grids for a given number of processors.

The original overset grids are read in PLOT3D[3] format instead of the PEGSUS format of Option 1. The subgrids of each separate grid are overlapped one grid cell by default. The overlap extent can be changed in the code internally.

2) BREAKUP PLOT3D MULTI-BLOCK GRIDS; OVERLAP SUBGRIDS

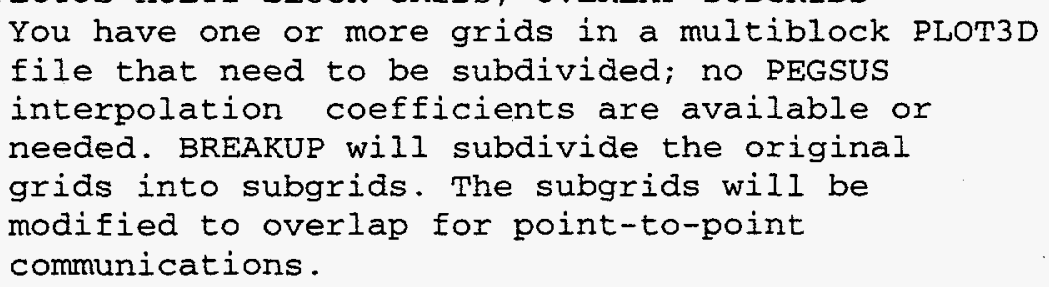

\section{c) Option 3 - PLOT3D-formatted Multiple Grids, No Interpolation Coefficients, No Subgrid Overlap Enforced; or Internally Generated Grid}

This option includes Option 2 but without the overlap. It also allows the user to run BREAKUP as a stand-alone code without the need for externally generated grids. Suboption a) internally generates a cubed grid. The user can then input any number of 
processors to divide the cube into the specified number of subgrids. This conveniently allows experimentation with the code 'right out of the box.' Suboption b) requires an externally generated PLOT3D-formatted file.

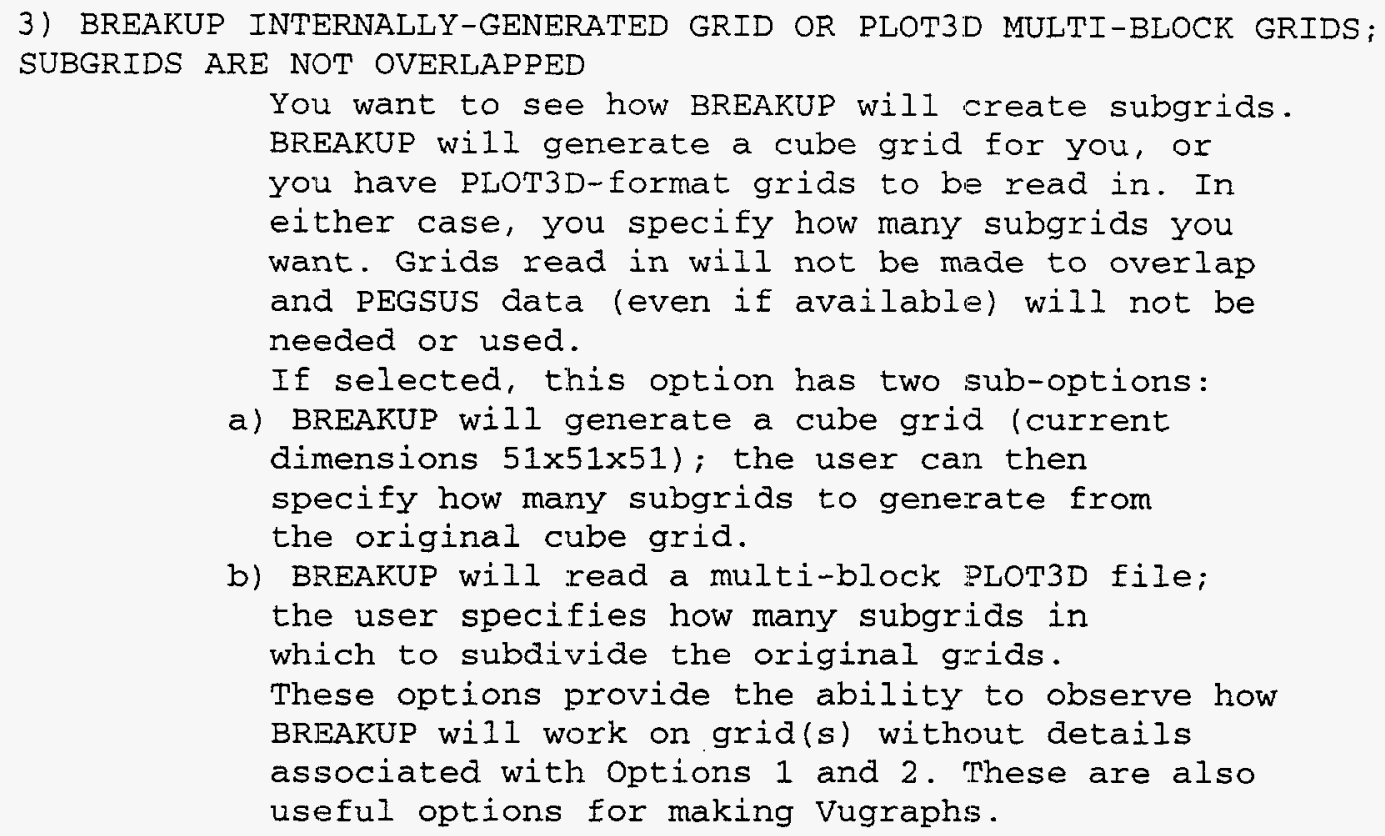

\section{Input Files}

The options discussed above require either PEGSUS files or PLOT3D grid files for input to BREAKUP. Particulars of these formats are listed below.

PEGSUS output (see User Option 1, above) used as input to BREAKUP consists of the following files.

1. INTOUT -interpolation coefficient file

2. COMPOUT -composite (overset) grid file

3. IBPLOT -iblank file

PLOT3D formatted files (see User Options 2 and $3 \mathrm{~b}$, above) must be named as follows for BREAKUP to read the files. The local directory is searched for the files in the order given. Hence, if the user has one grid file named XY.FMT (file-read sequence \#3, below) and another named G.FMT (file-read sequence \#9, below), BREAKUP will read only the former. BREAKUP is programmed to determine the file format (e.g., formatted, binary, multiple grids, etc.) automatically.

1. X.FMT

2. X.DAT

3. XY.FMT

4. XY.DAT

5. XYZ.FMT

6. XYZ.DAT 

7. GRID.FMT
8. GRID.DAT
9. G.FMT
10. G.DAT

\section{Output Files}

Output files from BREAKUP are listed below with brief explanations. Not all may be output during a run session since the particular options to do so may not be chosen by the user.

1. UNRAVELED

2. BREAKUP.OUT

3. POINTS_OUT

4. MESH_OUT

5. 3D_OVERSET_TABLE

6. 2D_OVERSET_TABLE

7. GRIDS2D

8. GRIDS3D

9. 3D_PATCH_TABLE

10. 2D_PATCH_TABLE

11. GRID_ORIGINAL.G

12. GRID_DIVIDED.G

13. 2Dxxxx.FMT

14. 3Dxxxx.FMT main output file to show user what BREAKUP did condensed version of screen output

PLOT3D file for interpolation and boundary points PLOT3D file for the 'iblank'ed mesh

file containing base (points used for interpolation) and target zones (points to be interpolated), indices, interpolation coefficients

decimated version of file 3D_OVERSET_TABLE for two-dimensional problems PLOT3D file for all 2-D grids or subgrids PLOT3D file for all 3-D grids or subgrids table of 3-D grid overlap links between subgrids of an overset grid table of 2-D grid overlap links between subgrids of an overset grid

Original grid file in subroutine PLOT3D file of subgrids generated by BREAKUP $2 D$ subgrid files where each subgrid is written to a separate file; $x x x x=0000$ to 9999

$3 \mathrm{D}$ subgrid files where each subgrid is written to a separate file; $\mathrm{xxxx}=0000$ to 9999

\section{Examples}

The first example showing the utility of BREAKUP is presented in Fig. 6. A cube was internally generated and arbitrarily subdivided into 74 subgrids. The plot corresponds to the output of User Option 3a, discussed above.

Another example demonstrating the power of BREAKUP is illustrated in Fig. 7. The five grids generated around a multi-element airfoil are subdivided by BREAKUP into 16 grids for use on the corresponding number of processors. The original grids are presented in Fig. 7a and the divided grids in Fig. 7b. Presented in Fig. 7c is a close-up view of the solution. Smooth streamlines, even in recirculating regions, indicate that message passing between subgrids is behaving as expected. Note from this figure that BREAKUP did not 
subdivide the original grid around the slat. BREAKUP determined that the number of grid points for this grid were too few to subdivide further, given the number of userspecified processors. Parallel solutions for the airfoil were generated using a modified version of INS2D[4-6]. Plots were generated using TECPLOT[7].

The next example, shown in Fig. 8, is the for an F-16 forward fuselage and intake duct as obtained from Lockheed-Martin. Presented in Fig. 8a are the original 19 grids. Ilustrated in Fig. $8 \mathrm{~b}$ are the results of subdividing the original 19 grids into 32 subgrids.

The last example, Figs. 9-11, involves two-dimensional ocean current solutions on coastal grids for the Gulf of Mexico and Greater Antilles islands. None of the solutions were run to convergence since this representation is non-physical. The main objective was to determine whether BREAKUP was properly assigning subgrid interpolation coefficients to the processors. The original grids are presented in Fig. 9a. The solution on the original grids is presented in Fig. 9b-d showing velocity vectors, a close-up of the velocity vectors around $\mathrm{Cuba}$, and streamlines, respectively. The original grids were then subdivided into a total of 16 subgrids as presented in Fig. 10a. As expected, BREAKUP subdivided the grids so that approximately the same number of grid points would reside on each processor. A close-up solution for the velocity vectors around Cuba is shown in Fig. 10b. Finally, the original grids were divided into 100 subgrids as shown in Fig. 11a. A close-up velocity vector plot is presented in Fig. 11b. All indications are that, regardless of the number of processors, BREAKUP is generating correct connectivity tables for use in parallel computers. The sample BREAKUP output listed in Appendix C was generated for one of the Gulf simulations noted above.

\section{Future Work and Directions}

Any future work on BREAKUP should include coding to aid the user in specifying solver boundary conditions on the subgrids. Specifying boundary conditions by "cut-and paste" editing methods can be a daunting task when preparing overset grids for a multi-thousand processor computer.

Another area of research should be developing the capability within BREAKUP to determine which processors require the highest amount of true interpolated data and to locate these processors near or adjacent to each other.

Modifications should be made to BREAKUP to help implement solvers on: heterogeneous, clustered, parallel processors and shared-memory multi-processor workstations. There is a significant amount of work to be done in this area.

An interesting concept would be for BREAKUP to optimize its output based on each processor's configuration. BREAKUP would need to know or to determine a parallel configuration's processors, memories, bandwidths, latencies, cache capabilities, etc. Obviously, for this process to be automated, BREAKUP would need to run on a machine able to access all processors to be used in the computations. This would not always be possible. 
Commensurate with further BREAKUP development should be the task of modifying other overset-grid-based solvers to run on parallel computers and establishing visualization needs for parallel overset-grid solutions.

\section{Summary}

The proof of concept in generating high-performance, high-resolution solutions using overset grids in parallel solvers has been demonstrated. Use of overset grids on massively parallel computers will increase users' capabilities to solve challenging problems.

Overset grid technology appears to be well suited for a parallel environment. However, research needs to be conducted for more efficient implementation. This is particularly true due to the ever-changing paradigms and implementations of parallel computing. It is expected that this effort would benefit a wide range of applications important to US industry as well as government laboratories and academia. 


\section{References}

1. J. L. Steger, F. C. Dougherty, and J. A. Benek, "A Chimera Grid Scheme," Advances in Grid Generation, K. N. Ghia and U. Ghia, eds., ASME FED-Vol. 5, June 1983.

2. N. E. Suhs and R. W. Tramel, "PEGSUS 4.0 User's Manual," AEDC-TR-91-8, Calspan Corporation / AEDC Operations, Arnold AFB, Tennessee, USA.

3. P. Walatka, P. G. Buning, L. Pierce, and P. A. Elson, "PLOT3D User's Manual," NASA Technical Memorandum 101067, March 1990.

4. S. E. Rogers and D. Kwak, "An Upwind Differencing Scheme for the Steady-State Incompressible Navier-Stokes Equations," NASA TM 101051, November 1988. Published in Journal of Applied Numerical Mathematics, Vol. 8, 1991, pp. 43-64.

5. S. E. Rogers and D. Kwak, "An Upwind Differencing Scheme for the Time Accurate Incompressible Navier-Stokes Equations," AIAA Journal, Vol. 28, No. 2, February, 1990, pp. 253-262.

6. S. E. Rogers, D. Kwak, and C. Kiris, "Numerical Solution of the Incompressible Navier-Stokes Equations for Steady-State and Time-Dependent Problems, AIAA Journal, Vol. 29, No. 4, April 1991, pp. 603-610.

7. "TECPLOT - Interactive Data Visualization for Scientists and Engineers, Version 6.0 User's Manual," Amtec Engineering, Inc., P.O. Box 3633, Bellevue, WA. 


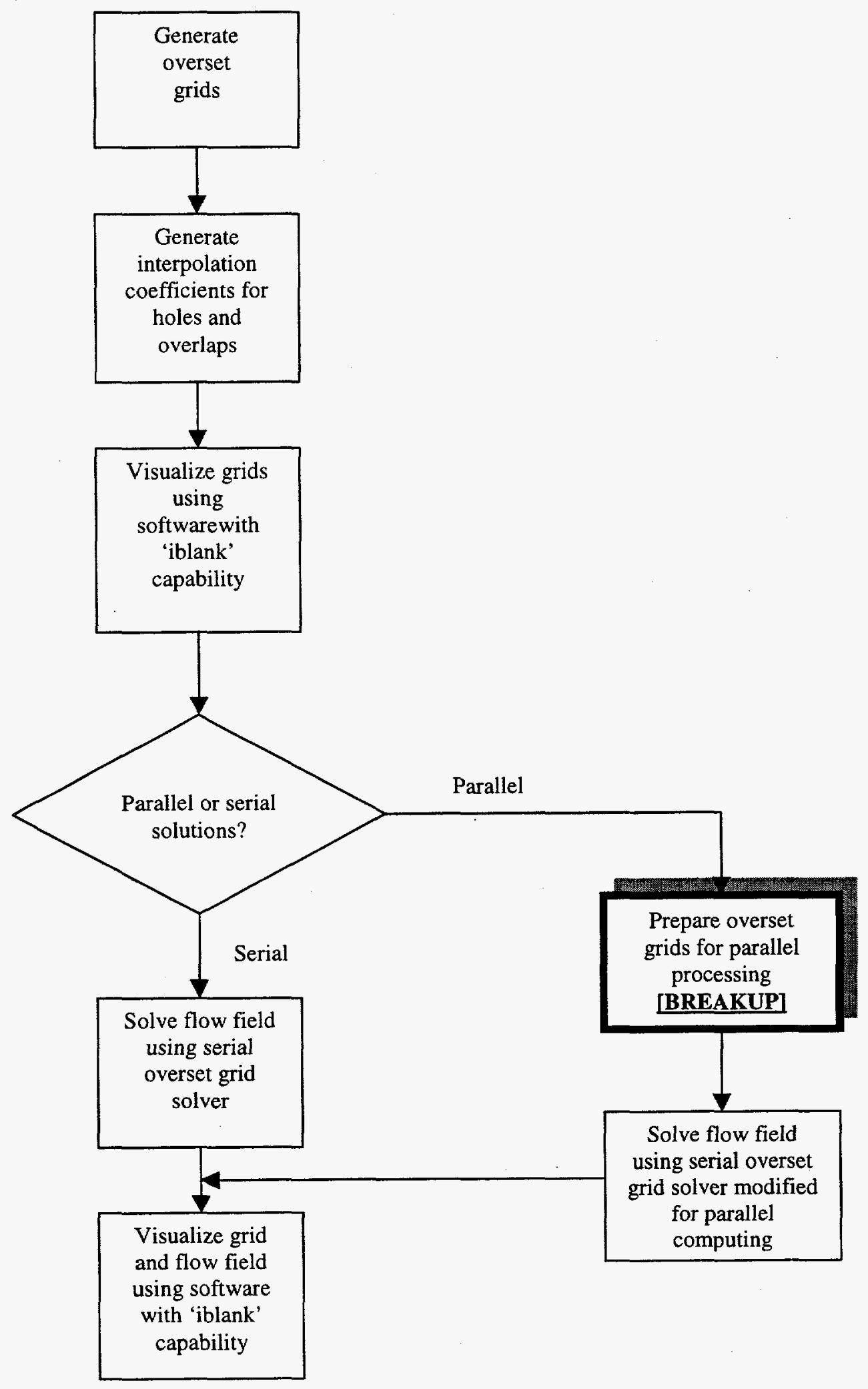

Figure 1. Overview flow chart for the parallel overset grid method. 


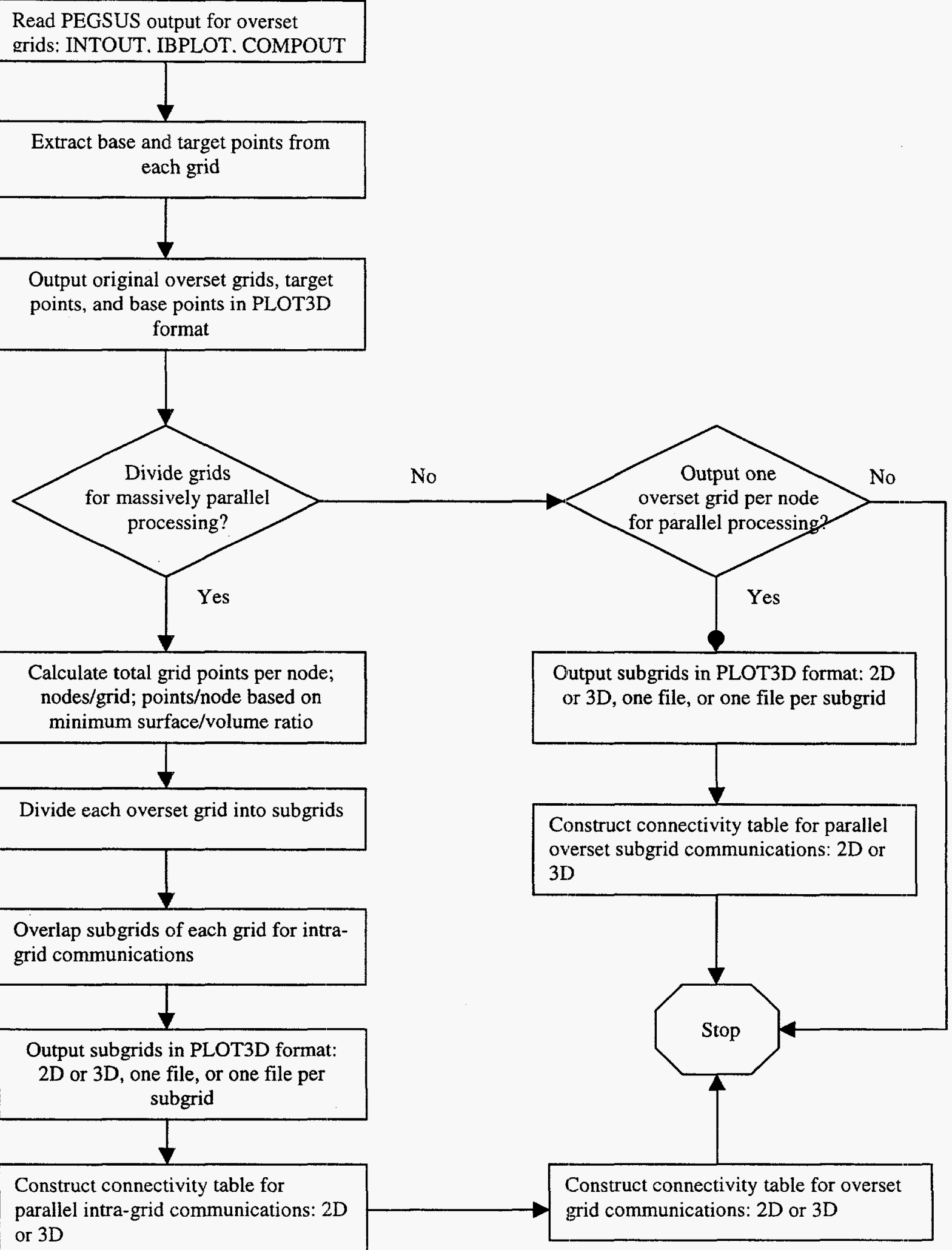

Figure 2. Overview flow chart for BREAKUP when overset grid output from PEGSUS is used as input. 
a) Subdivided Background Grid
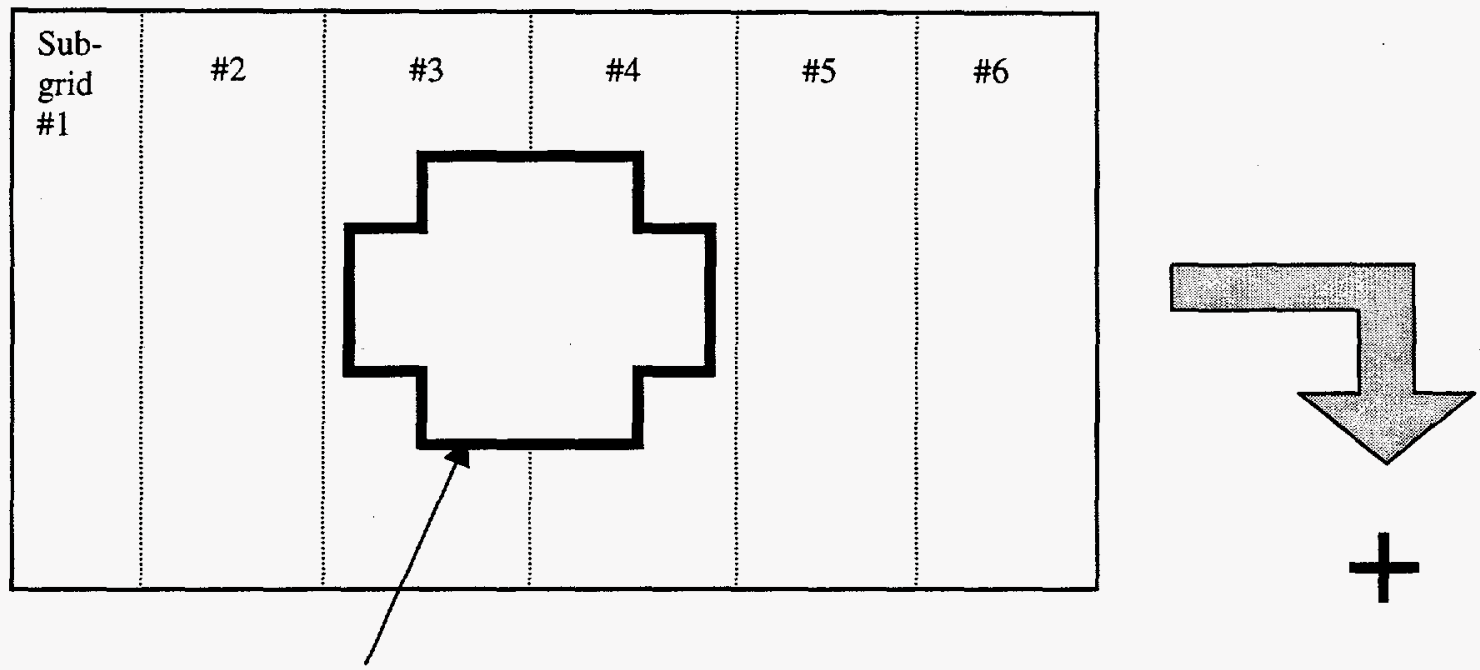

Background grid interior boundary (hole boundary) communicates with interior of geometry grid
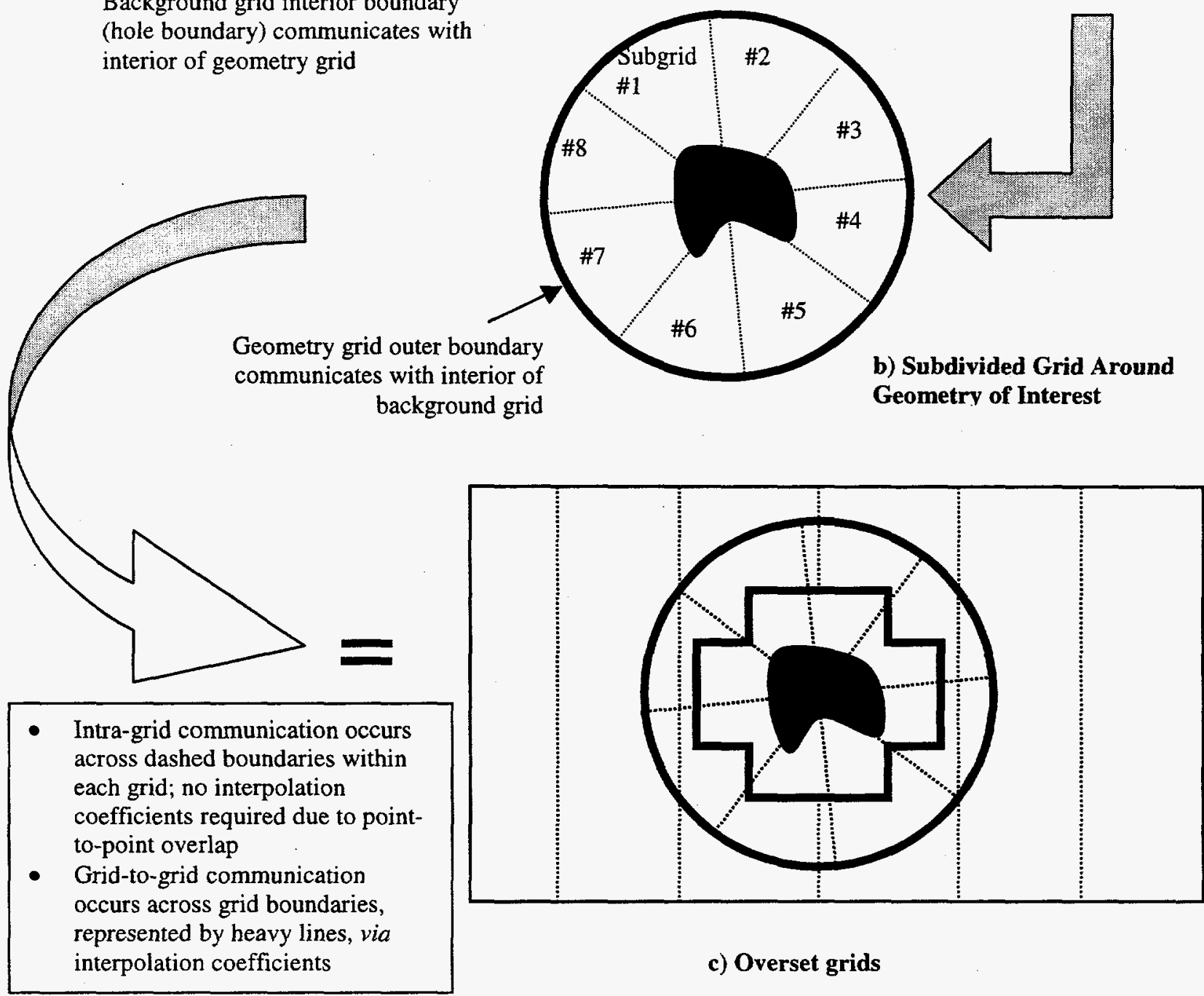

c) Overset grids

Figure 3. Illustration of increase in grid-to-grid and intra-grid communications when applying BREAKUP to overset grids. 


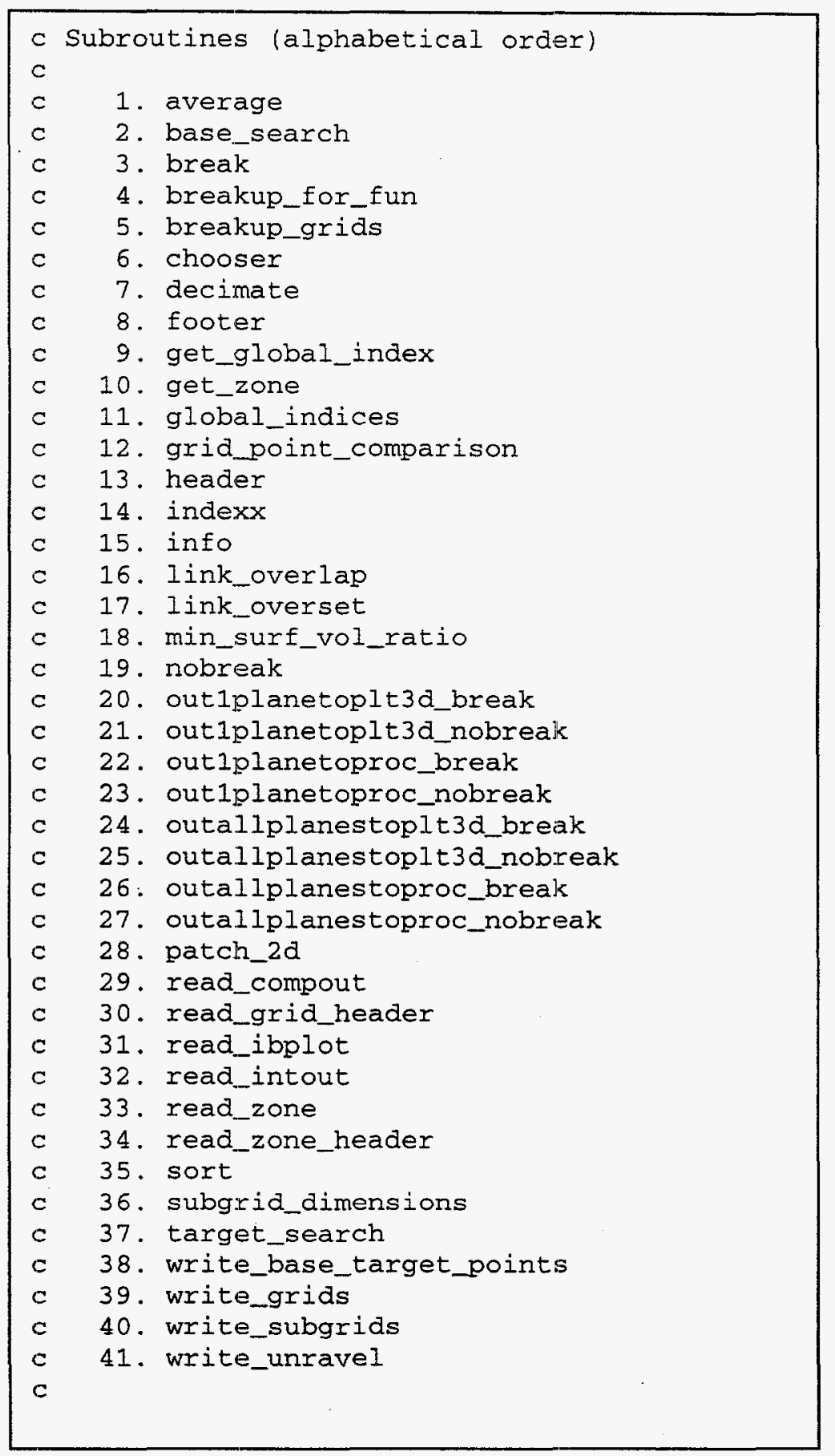

Figure 4. BREAKUP's subroutines listed in alphabetical order. 


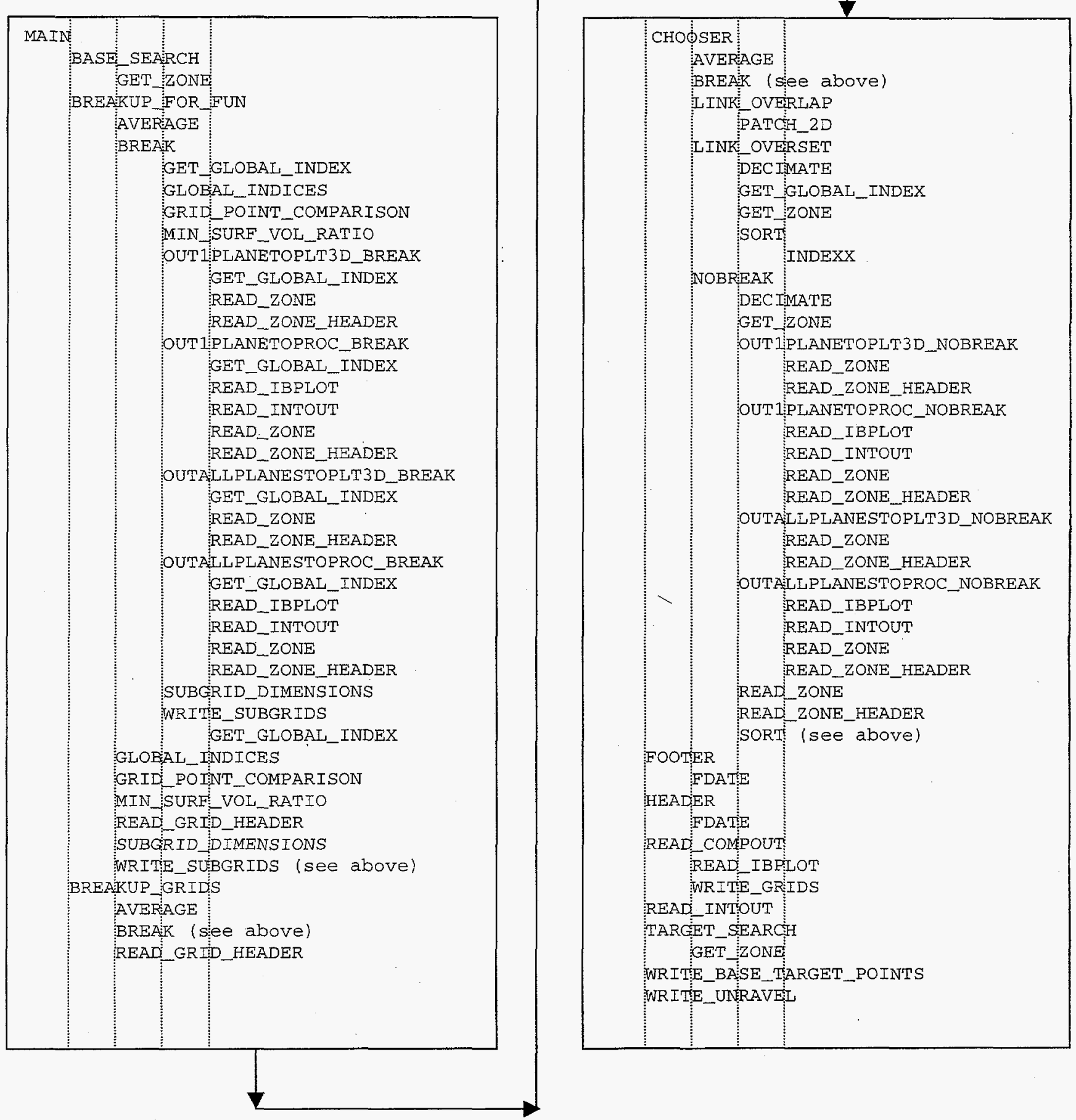

Figure 5. BREAKUP calling tree. 


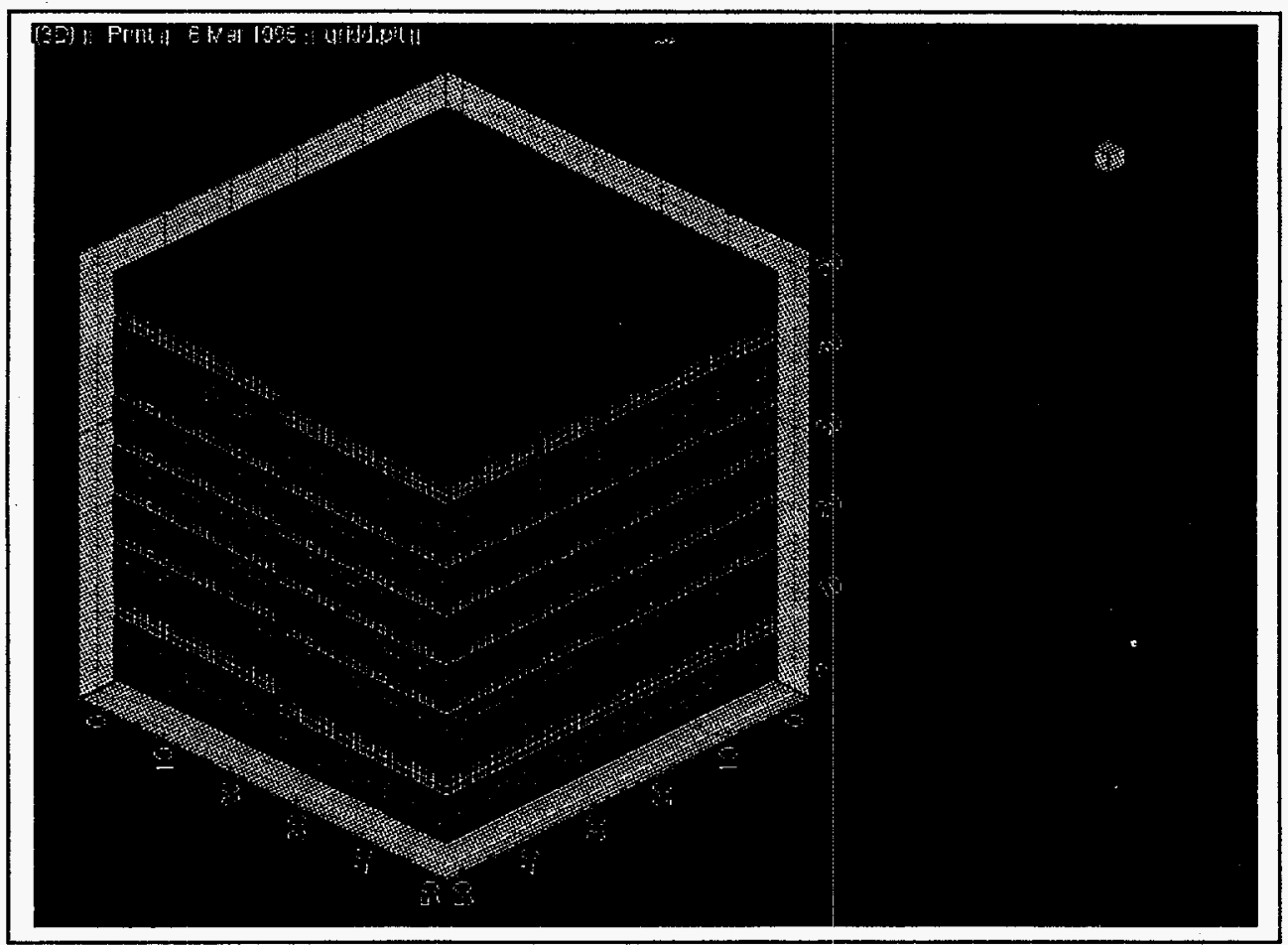

Figure 6. Cube illustrating how BREAKUP generates 74 subgrids. 


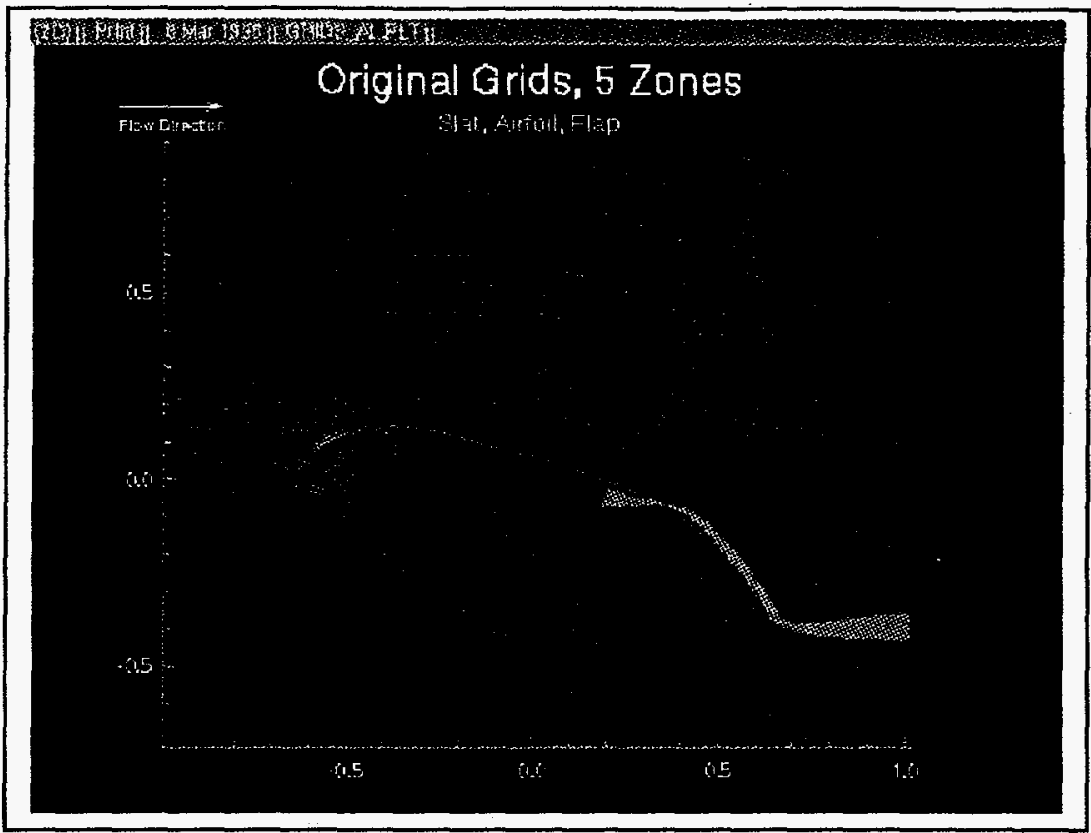

a) Original grids

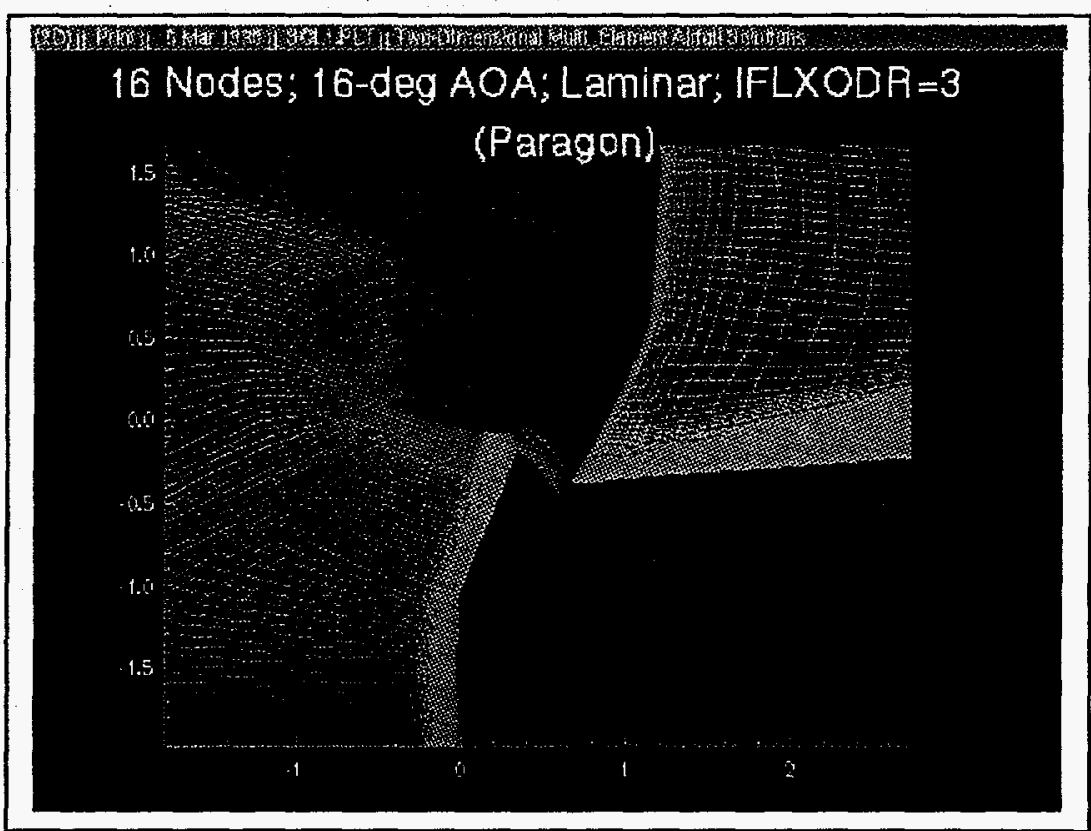

h) 16 suhgrids from BRF,AKITP

Figure 7. BREAKUP-generated subgrids for a multi-element airfoil grid, with solution. 


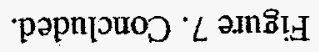

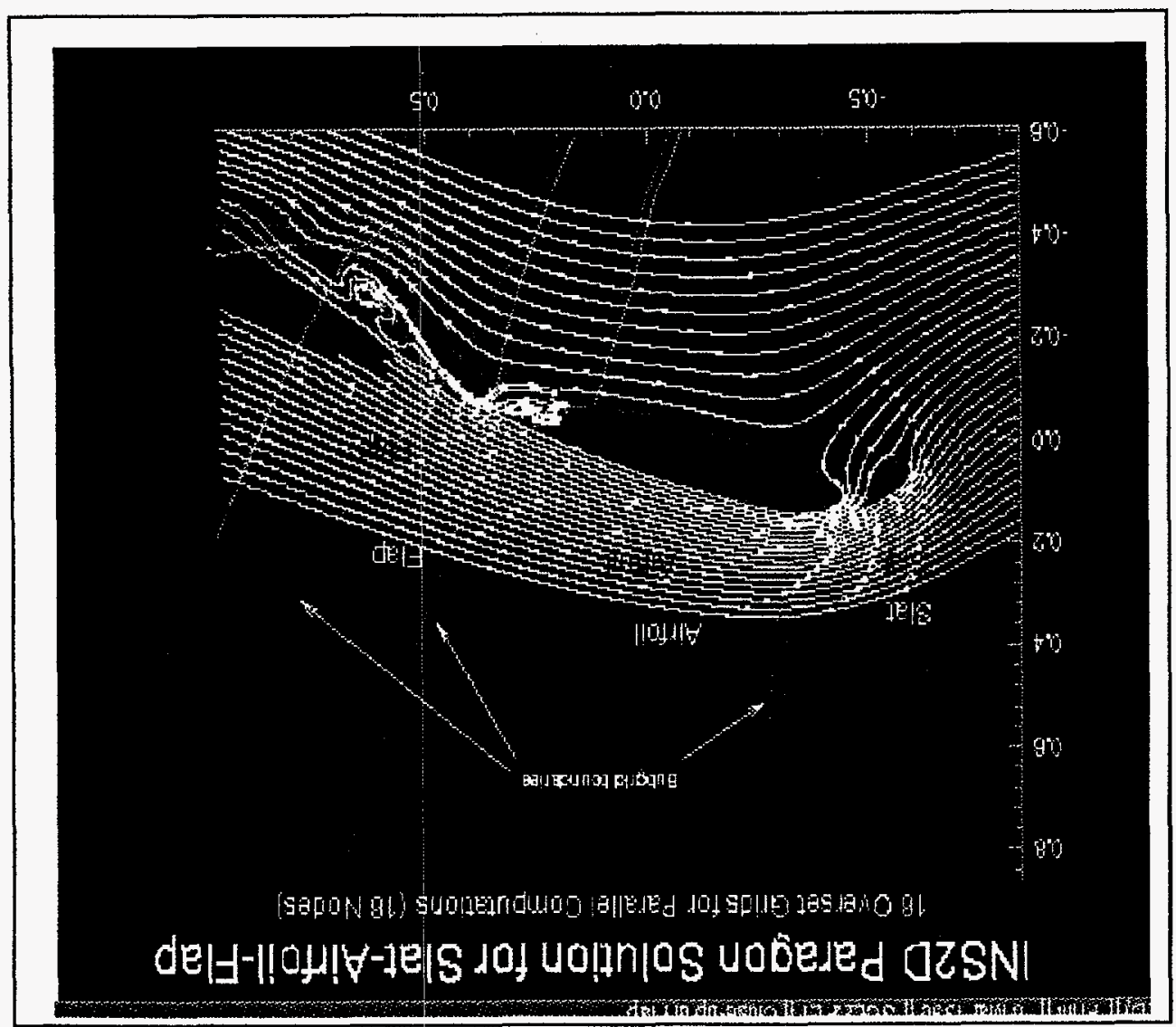


19 Original Grids from Lockheed-Martin for F16

Forward Fuselage and Intake Duct

a) 19 original grids

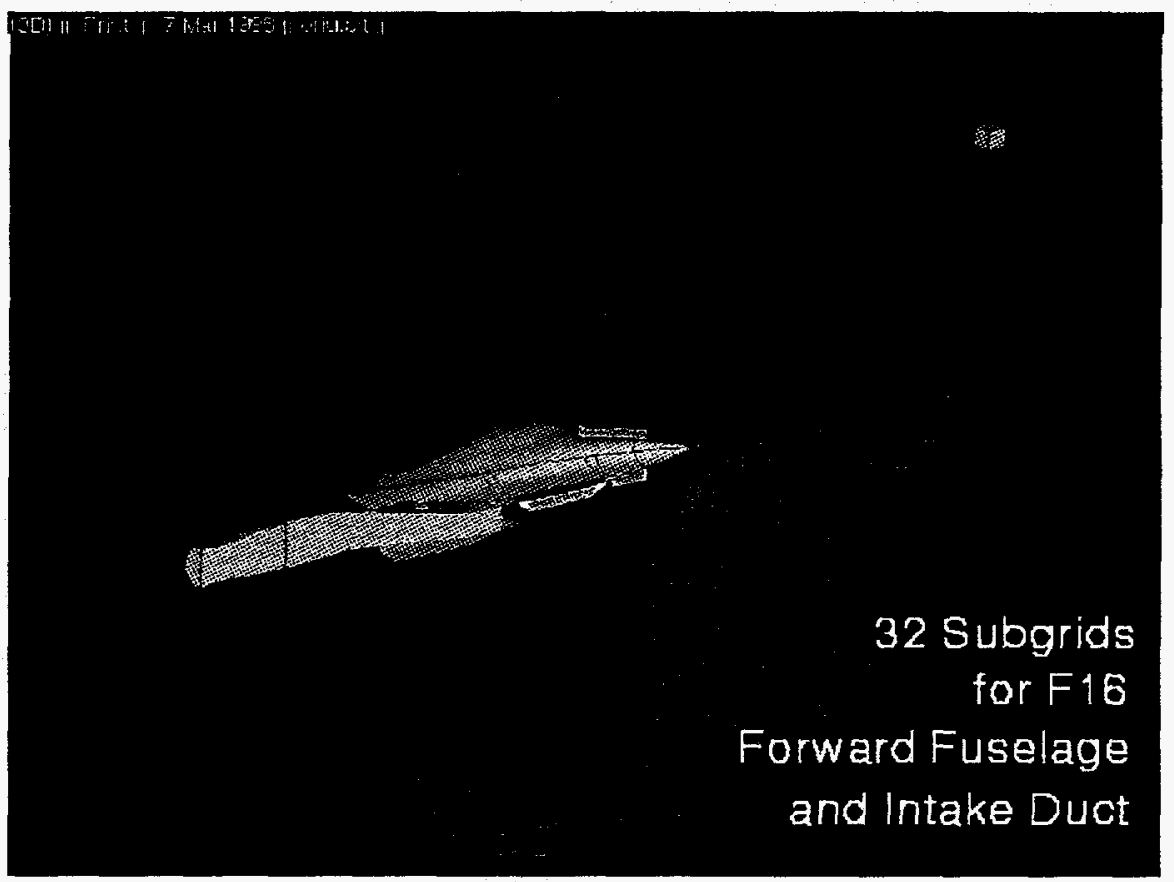

b) 32 subgrids generated by the BREAKUP code

Figure 8. BREAKUP subgrids for an F16's forward fuselage and intake duct . 


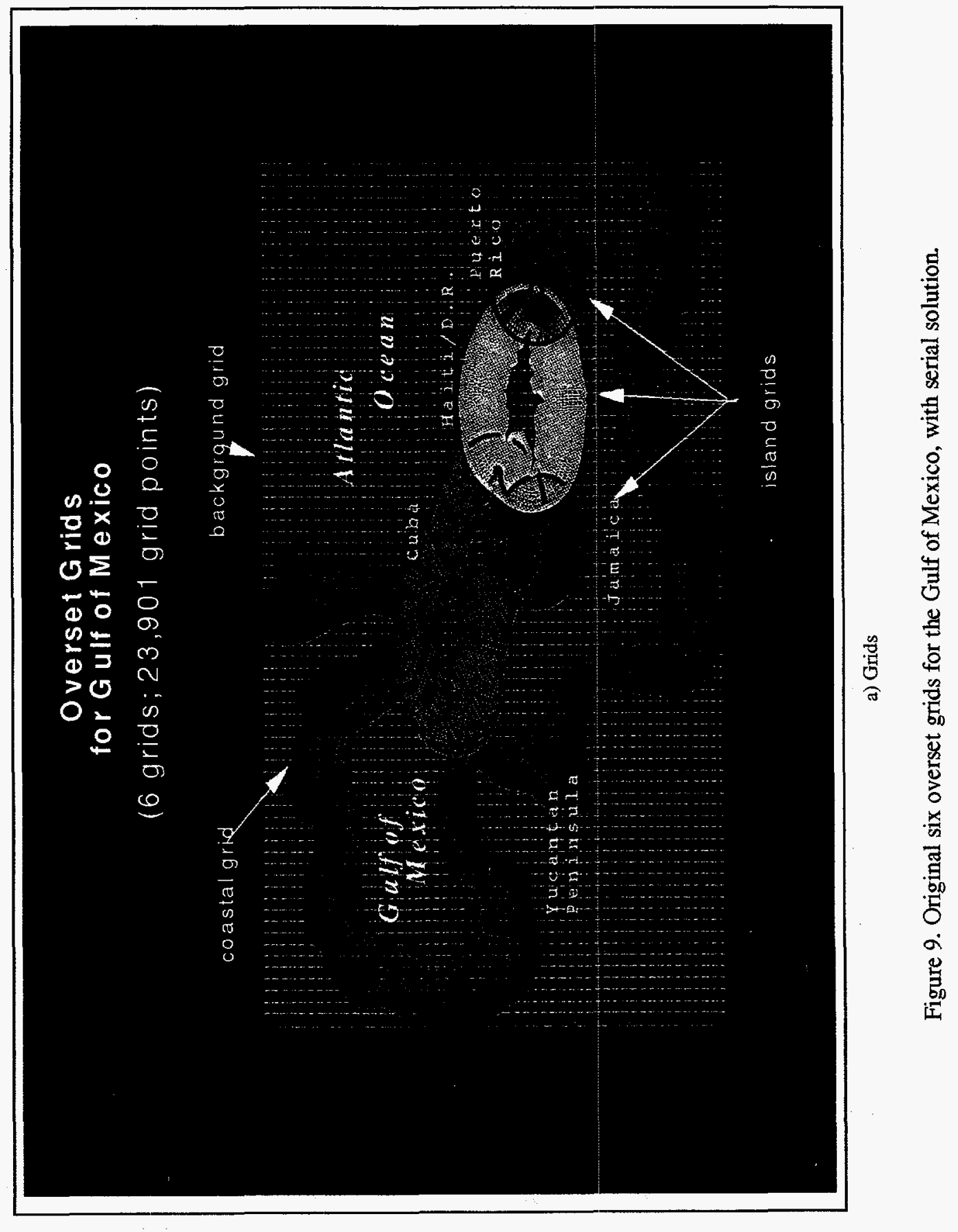




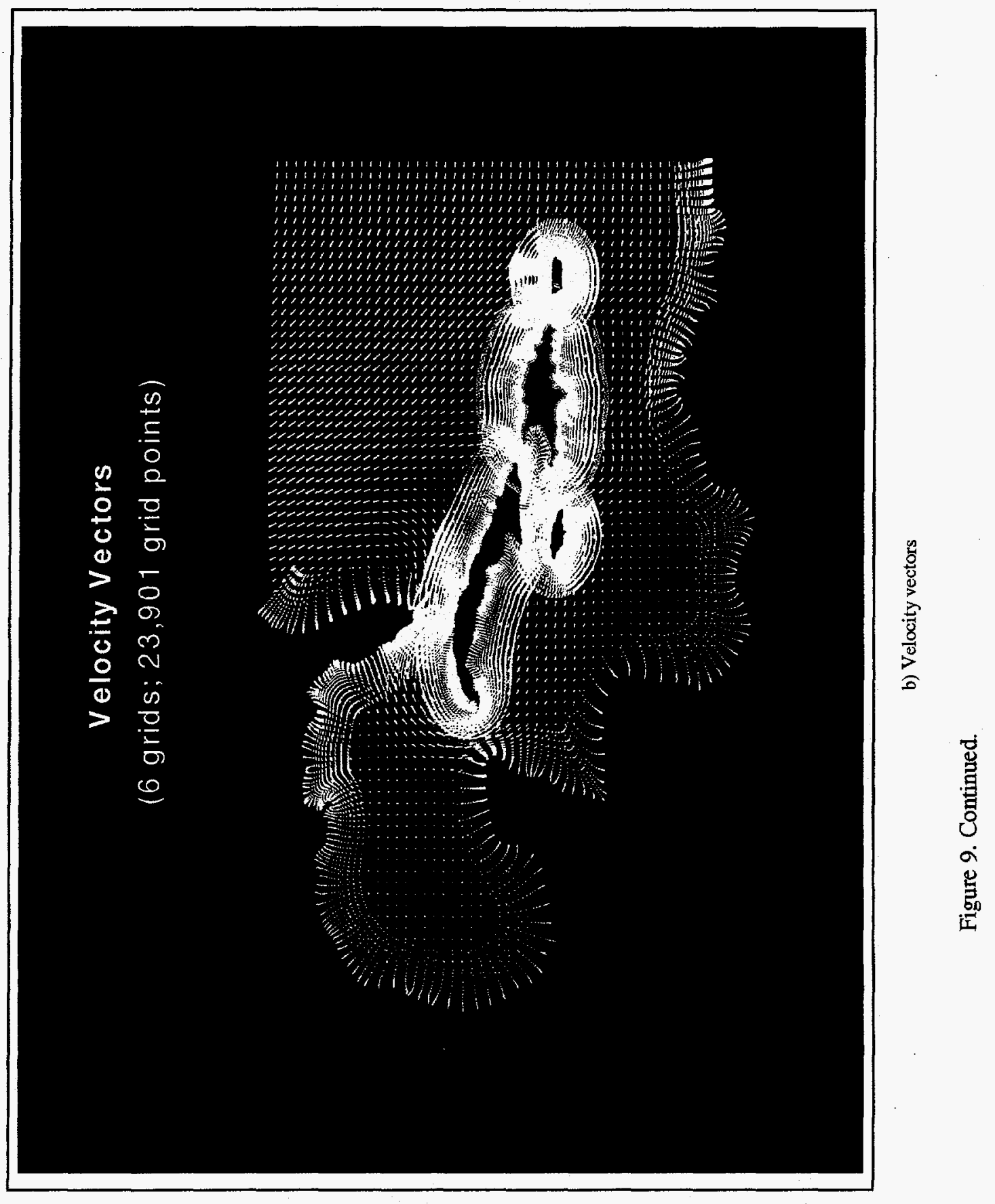




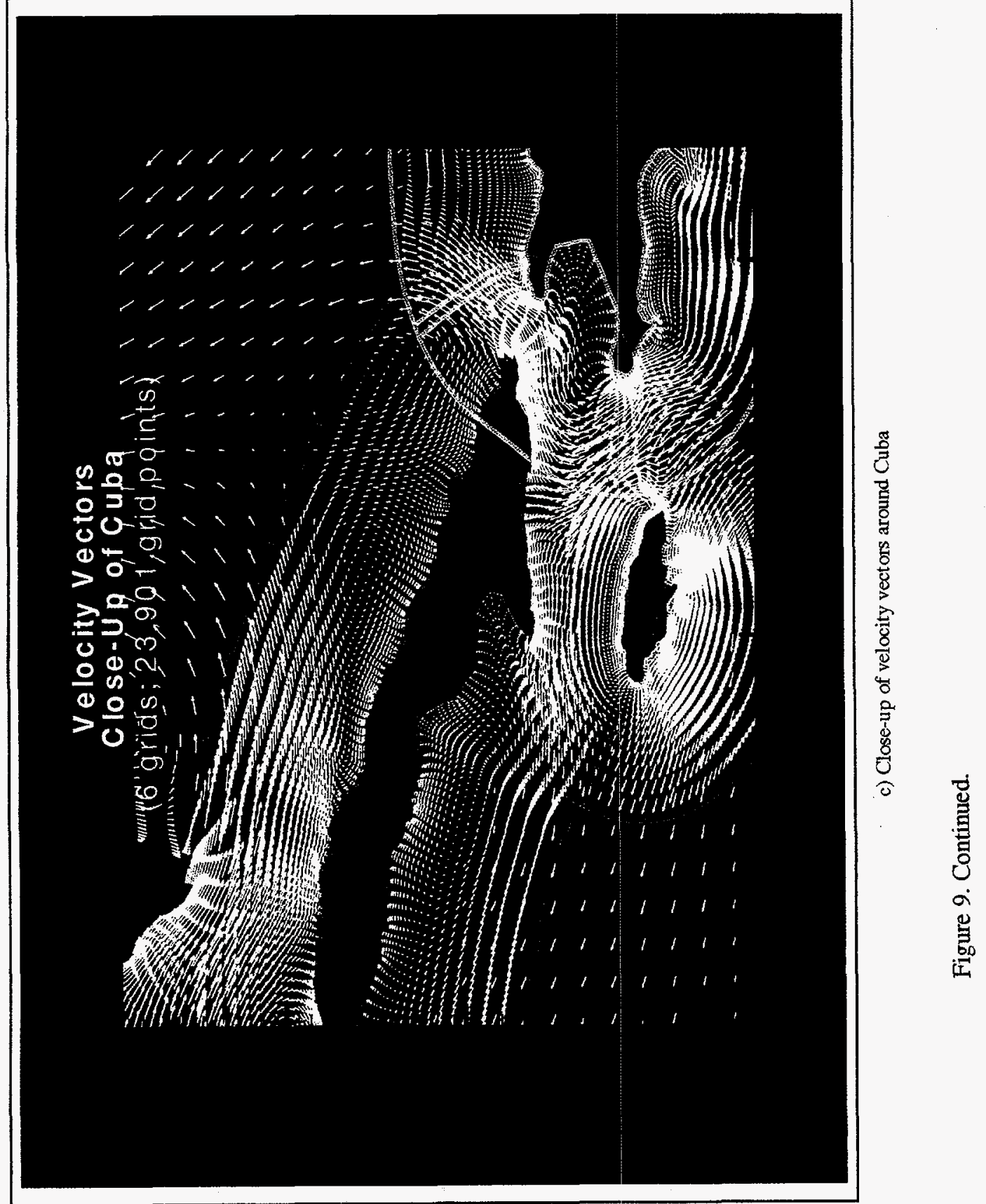




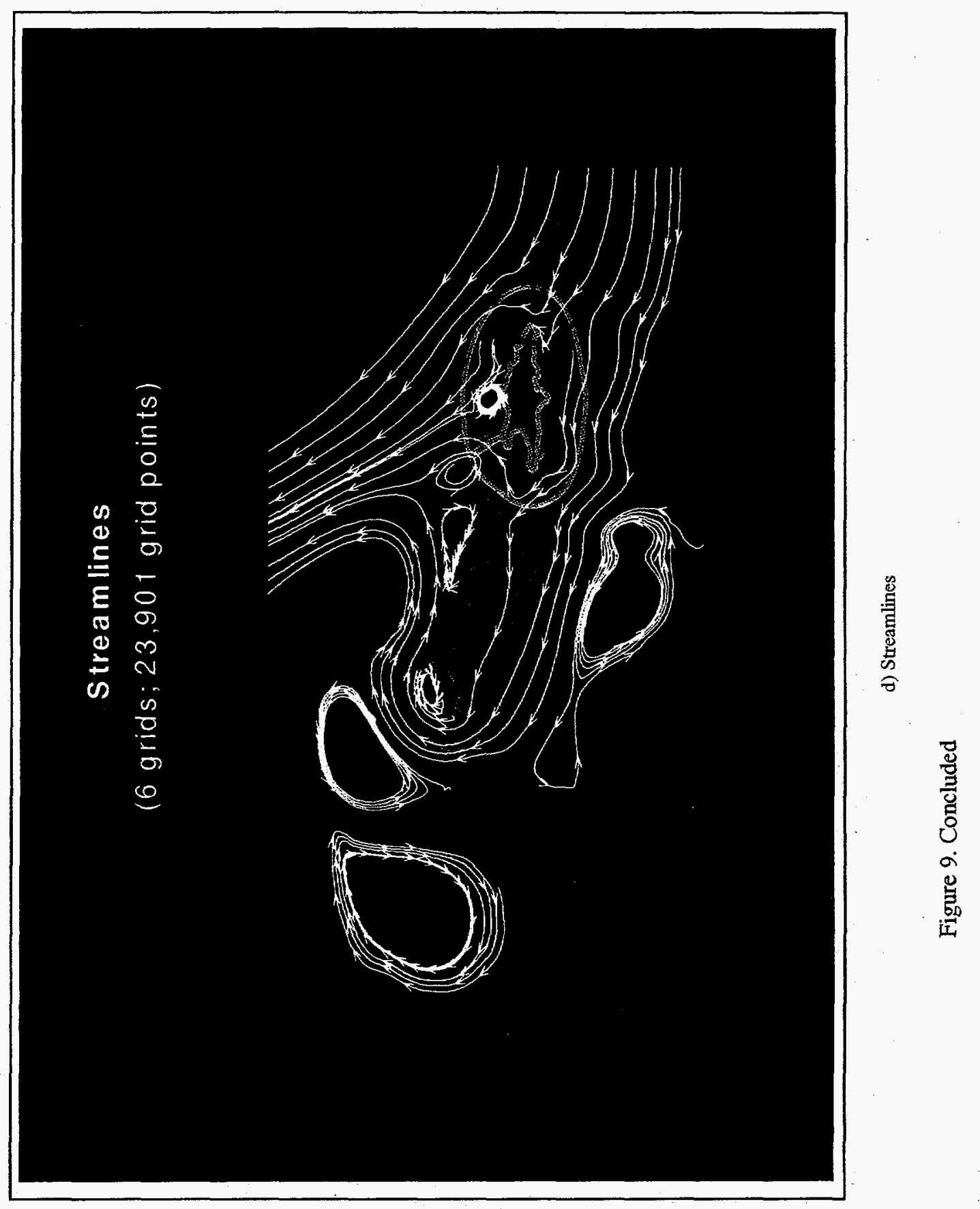




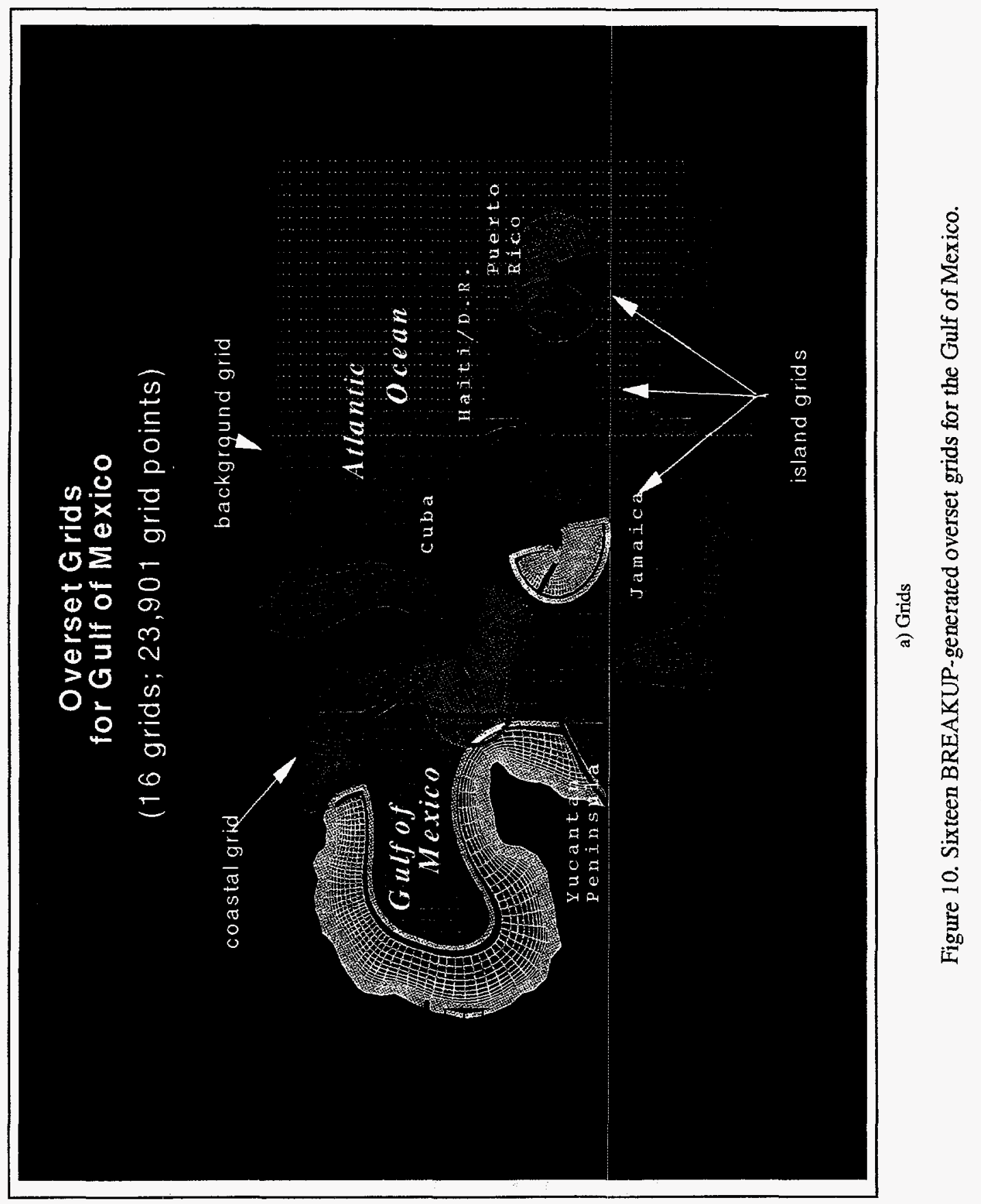




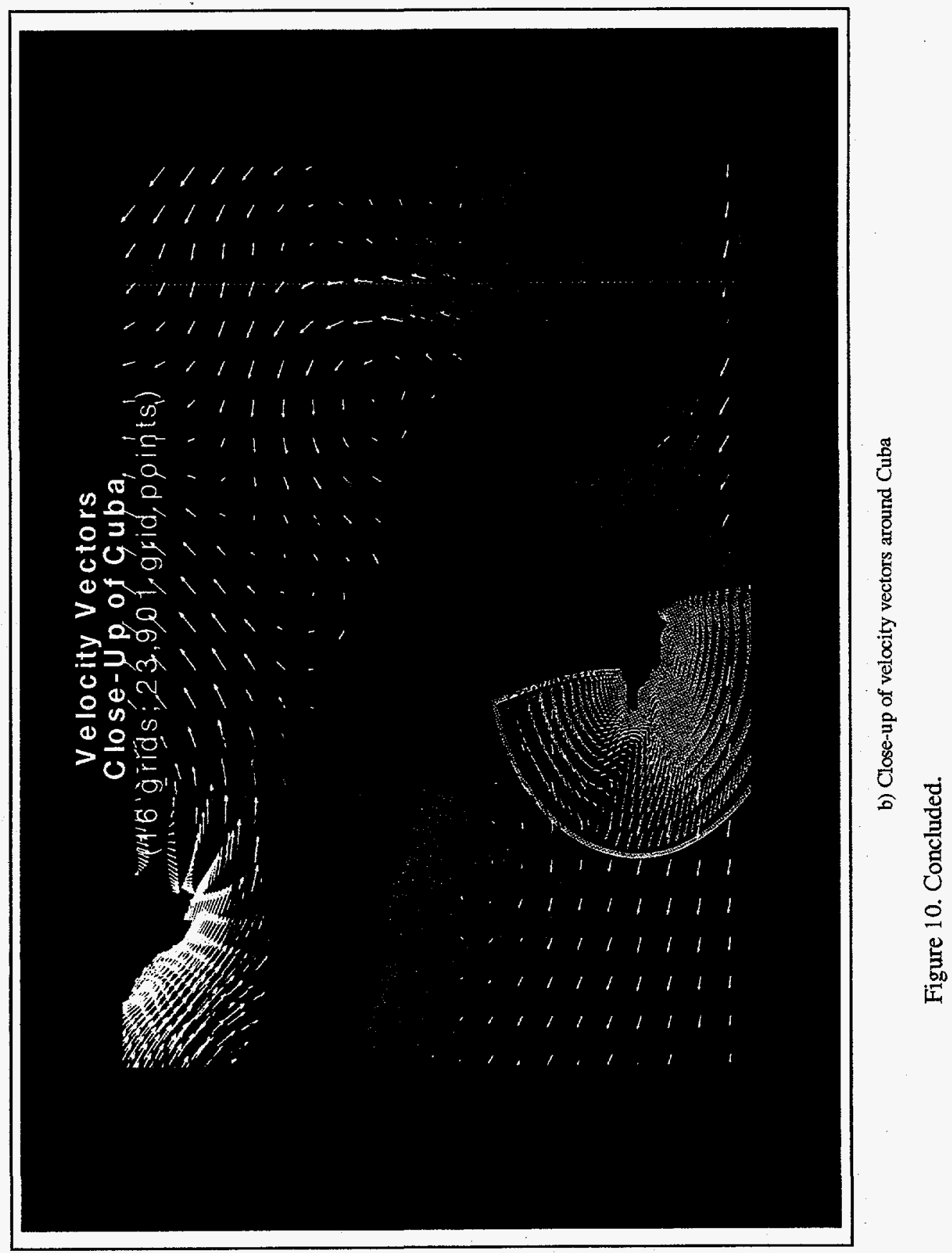




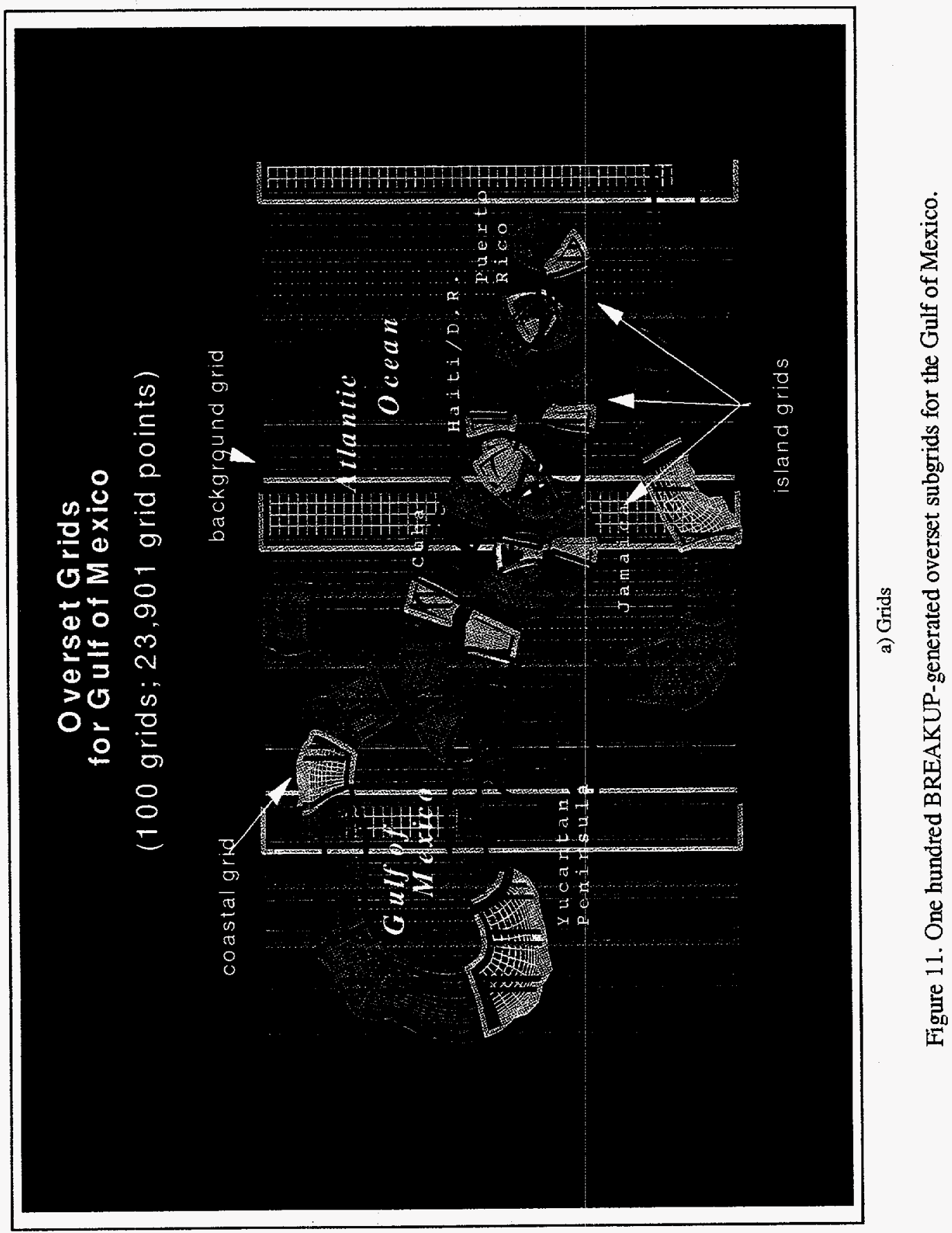




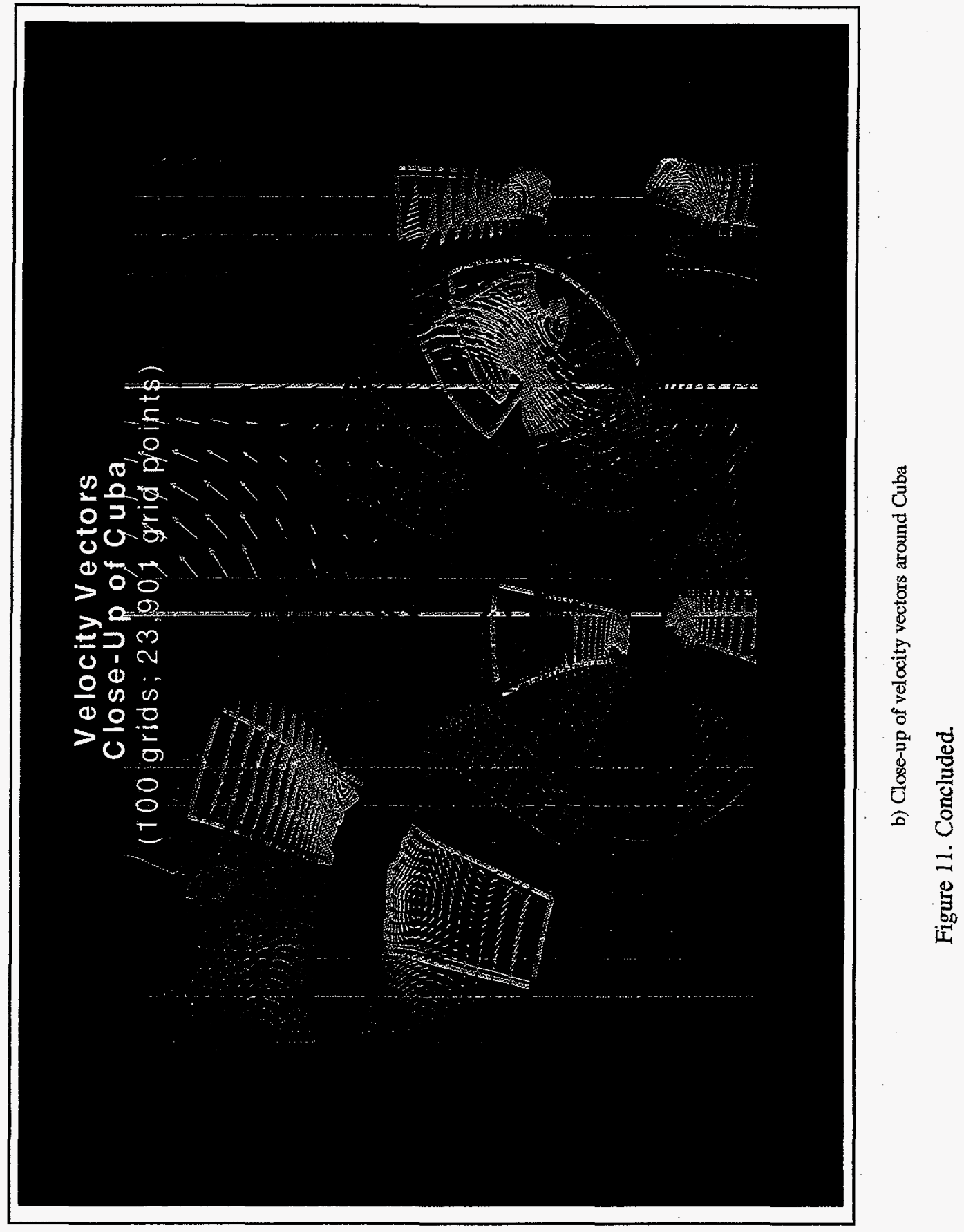




\section{Appendix A}

\section{BREAKUP Subroutines and Their Description}

Listed below in alphabetical order are the subroutines comprising BREAKUP. A brief description is given of each, along with the list of calling routines and the routines that are called.

\section{1. average}

Purpose:

This subroutine takes in all grids and computes average number of grid points per grid.

Calling routines:

breakup_for_fun

breakup_grids

chooser

Called routines, in order of appearance:

NONE

\section{2. base search}

Purpose:

This subroutine searches for base points for overset Chimera-

type grids

Calling routines:

main

Called routines, in order of appearance:

get_zone

get_zone

\section{3. break}

Purpose:

This subroutine breaks up grids into a user-specified number of zones.

Calling routines:

breakup_for_fun

breakup_grids

chooser

Called routines, in order of appearance:

min_surf_vol_ratio

subgrid_dimensions

grid_point_comparison

global_indices

get_global_index

write_subgrids 


$$
\begin{aligned}
& \text { get_global_index } \\
& \text { write_subgrids } \\
& \text { out 1planetoproc_break } \\
& \text { outallplanestoproc_break } \\
& \text { out1planetoplt3d_break } \\
& \text { outallplanestoplt3d_break }
\end{aligned}
$$

\section{4. breakup_for_fun}

Purpose:

Main calling code for breaking up grids without PEGSUS interpolation coefficients and without enforcing overlapped regions.

Calling routines:

\section{MAIN}

Called routines, in order of appearance:

min_surf_vol_ratio

subgrid_dimensions

grid_point_comparison

global_indices

write_subgrids

read_grid_header

average

break

\section{5. breakup_grids}

Purpose:

Main calling code for breaking up grids without PEGSUS interpolation coefficients; overlapped regions are generated between subgrids.

Calling routines:

\section{MAIN}

Called routines, in order of appearance:

read_grid_header

average

break

\section{6. chooser}

Purpose:

This subroutine gives the user the option to break up grids for parallel processing.

Calling routines:

$$
\text { MAIN }
$$

Called routines, in order of appearance: 

average
break
link_overlap
link_overset
nobreak

\section{7. decimate}

Purpose:

This subroutine reads data from file $>3$ D_OVERSET_TABLE $<$ (unit 15) and constructs file $>2$ D_OVERSET_TABLE $<$ by searching on the target $\mathrm{jkl}$ 's from file $>3 \mathrm{D} \_O V E R S E T$ TABLE $<$ and extracting only those with a to-be-calculated target index.

Calling routines:

link_overset

nobreak

Called routines, in order of appearance:

NONE

\section{8. footer}

Purpose:

This subroutine signs off.

Calling routines:

MAIN

Called routines, in order of appearance:

fdate

\section{9. get_global_index}

Purpose:

This subroutine is called to give the index range for subgrids using global indices. It is called from various locations throughout the code.

Calling routines:

break

link_overset

out1planetoplt3d_break

out1planetoproc_break

outallplanestoplt3d_break

outallplanestoproc_break

write_subgrids

Called routines, in order of appearance:

NONE 


\section{0. get_zone}

Purpose:

This subroutine reads in the proper zone ' $n z$ ' from

the grid file in any order

Calling routines:

$$
\begin{aligned}
& \text { base_search } \\
& \text { link_overset } \\
& \text { nobreak } \\
& \text { target_search }
\end{aligned}
$$

Called routines, in order of appearance:

NONE

\section{1. global_indices \\ Purpose:}

This subroutine sets up the global indices needed to break up the zone. Call this routine after calling subroutine subgrid_dimensions.

Calling routines:

break

breakup_for_fun

Called routines, in order of appearance:

NONE

\section{2. grid_point_comparison}

Purpose:

This subroutine calculates the total number of points for the original grid, and the total number of grid points for the subgrids of the original grid, and compares the two. The program stops if there is a difference. This is to provide some error checking.

Calling routines:

break breakup_for_fun

Called routines, in order of appearance:

NONE

\section{3. header}

Purpose:

This subroutine prints out the header for output.

Calling routines:

\section{MAIN}

Called routines, in order of appearance:

fdate 


\section{4. indexx}

Purpose:

Subroutine INDEXX indexes an array $\operatorname{iarr}(1: n)$, i.e., outputs the array indx $(1: n)$ such that $\operatorname{iarr}(\operatorname{indx}(j))$ is in ascending order for $j=1,2, \ldots, N$. The input quantities $n$ and iarr are not changed.

Calling routines:

sort

Called routines, in order of appearance:

NONE

\section{5. info}

Purpose:

This subroutine lists variables of interest, filenames, etc., to aid the user in understanding the program.

Calling routines:

NONE

Called routines, in order of appearance:

NONE

\section{6. link_overlap}

\section{Purpose:}

This subroutine links overlapped (patched) subgrids in a zone. This subroutine is not called for overlapped regions for which interpolation coefficients have been generated. The subgrids are assumed to align point-to-point.

\section{Calling routines:}

chooser

Called routines, in order of appearance:

write_unravel

patch_2d

\section{7. link_overset}

Purpose:

This subroutine links grid subgrids for overlapped embedded grids.

Calling routines:

chooser

Called routines, in order of appearance:

get_zone

get_global_index 
get_global_index

get_zone

sort

decimate

\section{8. min_surf_vol_ratio}

Purpose:

This subroutine calculates the 3 -factors for a given number of subgrids, then calculates the surface to volume ratio for each set of 3-factors given the maximum j,k,l dimensions of each grid.

Calling routines:

break

breakup_for_fun

Called routines, in order of appearance:

NONE

\section{9. nobreak}

Purpose:

This subroutine keeps all grids as was output by the PEGSUS

code. File 3D_OVERSET_TABLE is generated listing all base and target grid points. Grids are output as one file or as multiple files, user choice. Essentially, output is for running a flow solver on a serial machine or for distributed computing, not for massively parallel applications on concurrent processors.

Calling routines: chooser

Called routines, in order of appearance:

read_zone_header

read_zone

out 1planetoproc_nobreak

outallplanestoproc_nobreak

out 1 planetoplt3d_nobreak

outallplanestoplt3d_nobreak

get_zone

get_zone

sort

decimate

\section{0. out1planetoplt3d_break}

Purpose:

The purpose of this subroutine is to write one plane of each subgrid to file GRIDS (UNIT 20) in PLOT3D format for graphics.

Calling routines:

Break 
Called routines, in order of appearance:

read_zone_header

read_zone

get_global_index

get_global_index

get_global_index

\section{1. out1planetoplt3d_nobreak}

Purpose:

The purpose of this subroutine is to write one plane of each

of the original grids to file GRIDS2D (UNIT 20) in PLOT3D format

for graphics.

Calling routines:

nobreak

Called routines, in order of appearance:

read_zone_header

read_zone

\section{2. out1planetoproc_break}

Purpose:

The purpose of this subroutine is to output one plane of data

for each processor on a massively parallel computer. Output is to file $2 \mathrm{D}$ FMT, where is $0000,0001,0002$, etc.

Calling routines:

break

Called routines, in order of appearance:

read_zone_header

read_zone

read_intout

read_ibplot

get_global_index

get_global_index

get_global_index

\section{3. out1planetoproc_nobreak}

Purpose:

The purpose of this subroutine is to output one plane of data for each processor on a massively parallel computer. Output is to file $2 \mathrm{D}$ .FMT, where is $000,001,002$, etc.

Calling routines:

nobreak

Called routines, in order of appearance:

read_zone_header 


$$
\begin{aligned}
& \text { read_zone } \\
& \text { read_intout } \\
& \text { read_ibplot }
\end{aligned}
$$

\section{4. outallplanestoplt3d_break}

Purpose:

The purpose of this subroutine is to write all planes of each subgrid to file GRIDS3D (UNIT 20) in PLOT3D format for graphics.

Calling routines:

break

Called routines, in order of appearance:

read_zone_header

read_zone

get_global_index

\section{5. outallplanestoplt3d_nobreak}

Purpose:

The purpose of this subroutine is to write all planes of each subgrid to file GRIDS3D (UNIT 20) in PLOT3D format for graphics.

Calling routines:

nobreak

Called routines, in order of appearance:

read_zone_header

read_zone

\section{6. outallplanestoproc_break}

Purpose:

The purpose of this subroutine is to output all planes of data for each processor on a massively parallel computer. Output is to file 3D_.FMT, where __ is $0000,0001,0002$, etc.

Calling routines:

break

Called routines, in order of appearance:

read_zone_header

read_zone

read_intout

read_ibplot

get_global_index

\section{7. outallplanestoproc_nobreak}

Purpose:

The purpose of this subroutine is to output all planes of data 
for each processor on a massively parallel computer. Output is to file $X Y$ .FMT, where is $0000,0001,0002$, etc.

Calling routines:

nobreak

Called routines, in order of appearance:

read_zone_header

read_zone

read_intout

read_ibplot

\section{8. patch_2d}

Purpose:

Reduces the table of 3D patched, i.e. point-to-point overlapped

grids, to a $2 \mathrm{D}$ table for 2D flow solvers.

Calling routines:

link_overlap

Called routines, in order of appearance:

NONE

\section{9. read_compout}

Purpose:

This subroutine reads data from file COMPOUT, as generated by PEGSUS code.

\section{Calling routines:}

MAIN

Called routines, in order of appearance:

read_ibplot

write_grids

30. read_grid_header

Purpose:

This routine attempts to find the grid file, a plot3d 3D type

file, and determines whether it is formatted or unformatted, single or multiple zone, and with or without an iblank array.

It reads in the dimensions of the grid and performs parameter checks, and leaves the grid file opened with the pointer after the records containing the dimensions.

Much of this subroutine taken from code written by Phil Stuart of NASA JSC.

Calling routines:

breakup_for_fun

breakup_grids

Called routines, in order of appearance:

NONE 


\section{1.read_ibplot}

Purpose:

This subroutine reads the IBPLOT array, as generated by

PEGSUS code, one zone at a time

Calling routines:

MAIN

outlplanetoproc_break

out 1planetoproc_nobreak

outallplanestoproc_break

outallplanestoproc_nobreak

read_compout

Called routines, in order of appearance:

NONE

32. read_intout

Purpose:

This subroutine reads the INTOUT file, as generated by the PEGSUS code, one zone at a time. It includes the IBLANK PEGSUS code array.

Calling routines:

MAIN

out lplanetoproc_nobreak

outallplanestoproc_break

outallplanestoproc_nobreak

Called routines, in order of appearance:

exit

exit

\section{3. read_zone}

Purpose:

This subroutine sequentially reads the grid file one zone at a time. The grid file read in by this subroutine is assumed to be a 3-d PLOT3D formatted multi-zone file. The read pointer has been positioned past the header data by previously calling subroutine read_zone_header.

Calling routines:

nobreak

out 1planetoplt3d_break

out 1 planetoplt3d_nobreak

out 1 planetoproc_break

out l planetoproc_nobreak

outallplanestoplt3d_break 
outallplanestoplt3d_nobreak

outallplanestoproc_break

outallplanestoproc_nobreak

Called routines, in order of appearance:

NONE

\section{4. read_zone_header}

Purpose:

This subroutine reads the grid file header and leaves the read pointer at the start of the grid points for the first

zone. The grid file read in by this subroutine is assumed to be a 3-d PLOT3D formatted multi-zone file.

Calling routines:

nobreak

out1planetoplt3d_break

out1planetoplt3d_nobreak

out1planetoproc_break

out 1planetoproc_nobreak

outallplanestoplt3d_break

outallplanestoplt3d_nobreak

outallplanestoproc_break

outallplanestoproc_nobreak

Called routines, in order of appearance:

NONE

35. sort

Purpose:

This subroutine reads data from scratch file SCRATCH25, sorts on the base processor, then sorts the target processor for each base processor, so a double sort is needed. Scratch file

SCRATCH 30 is used as a temporary scratch file between sorts.

Calling routines:

link_overset

nobreak

Called routines, in order of appearance:

indexx

index $x$

\section{6. subgrid_dimensions}

Purpose:

This subroutine calculates the dimensions of the subgrids.

Calling routines:

break 
breakup_for_fun

Called routines, in order of appearance:

NONE

37. target_search

Purpose:

This subroutine searches for target points for overset Chimeratype grids

Calling routines:

MAIN

Called routines, in order of appearance:

get_zone

get_zone

38. write_base_target_points

Purpose:

This subroutine writes base (stencil) and target (boundary) points for each zone in PLOT3D format.

Calling routines:

MAIN

Called routines, in order of appearance:

NONE

\section{9. write_grids}

Purpose:

This subroutine writes grids, read from PEGUS output, in

PLOT3D format. The data in this format is read in later

and subdivided into the user-specified number of subgrids.

Calling routines:

read_compout

Called routines, in order of appearance:

NONE

\section{0. write_subgrids}

Purpose:

This subroutine writes out the subgrids formed in subroutine

SUBGRID_DIMENSIONS and subroutine GLOBAL_INDICES.

Calling routines:

break

breakup_for_fun

Called routines, in order of appearance:

get_global_index 
41. write_unravel

Purpose:

This subroutine outputs to file UNRAVEL. Output depends on iflag.

Calling routines:

MAIN

link_overlap

Called routines, in order of appearance:

NONE 


\section{Appendix B}

\section{List of Variables used in BREAKUP}

Listed below are the more significant variables used in BREAKUP. A brief description is given of each. If not listed below, the variable's meaning is probably obvious from the context of the coding.

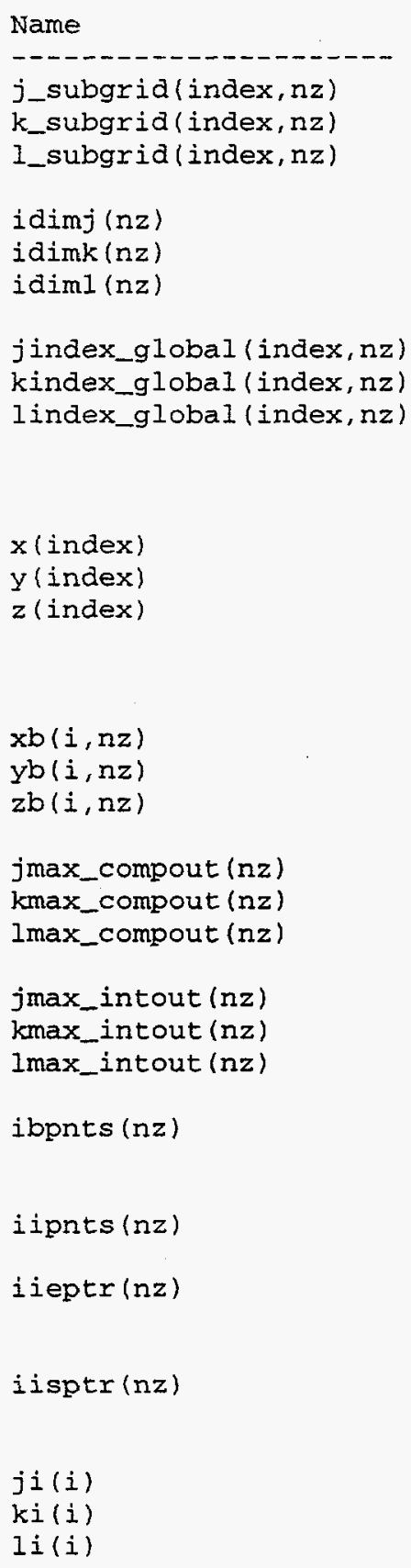

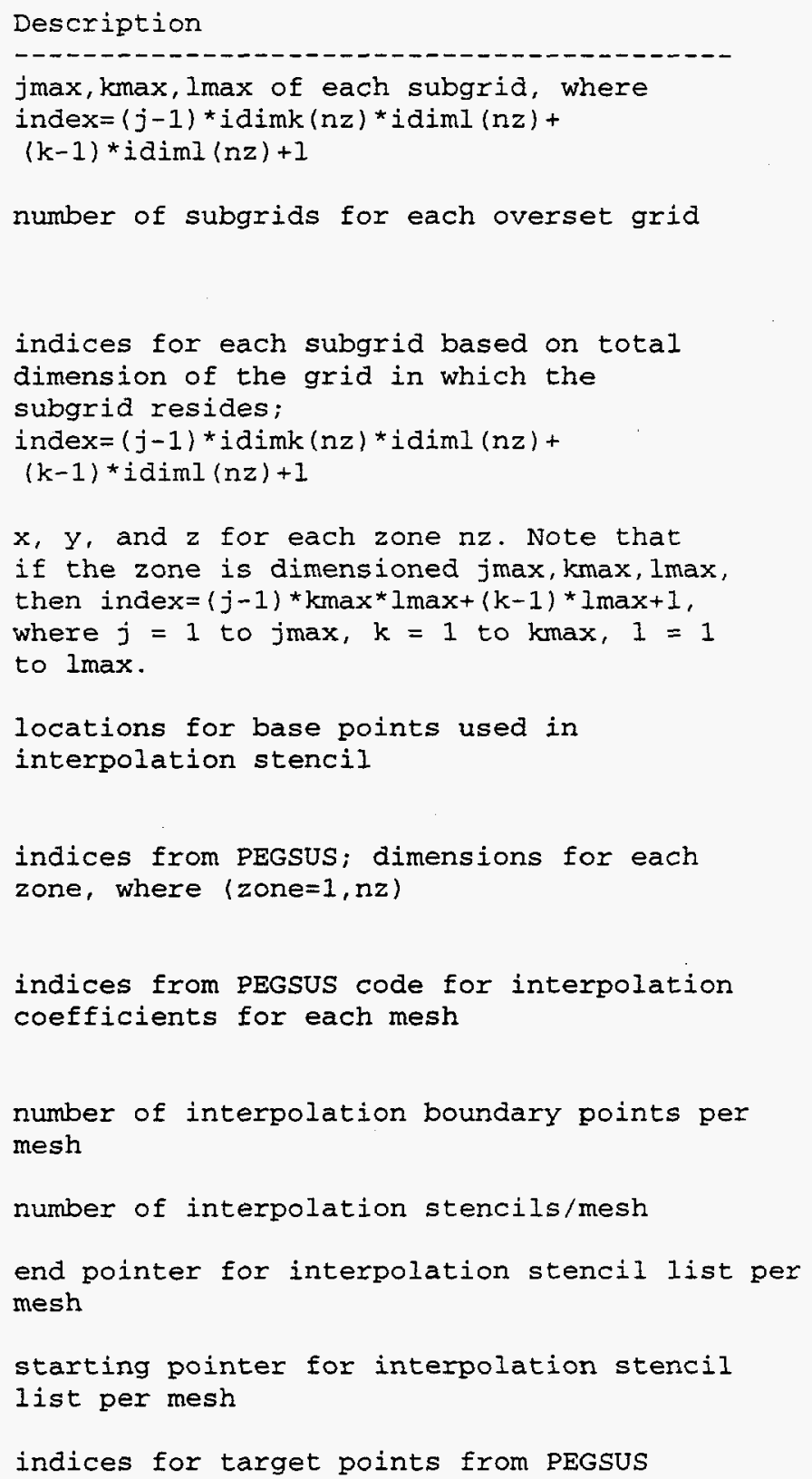


Name

- - - - - - - - - - - - - - - - - -

jb(i)

$\mathrm{kb}(i)$

$1 b(i)$

ibc

ibplot (index)

iblank (index)

$d x(i)$

dy (i)

$d z(i)$
Description

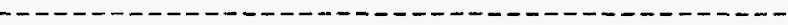

indices for base points from PEGSUS

interpolation boundary point pointer

array rea in from PEGSUS file IBPLOT and is the IBIANK array typically used in plotting PLOT3D Eiles

array read in from PEGSUS file INTOUT; used as a flag to the flow solver to tell it that particular boundary points are updated by PEGSUS interpolation and not by the solver

interpolation coefficients from PEGSUS 


\section{Appendix C}

\section{Sample Output from BREAKUP (file BREAKUP.OUT)}

Note: line numbers have been added for reference

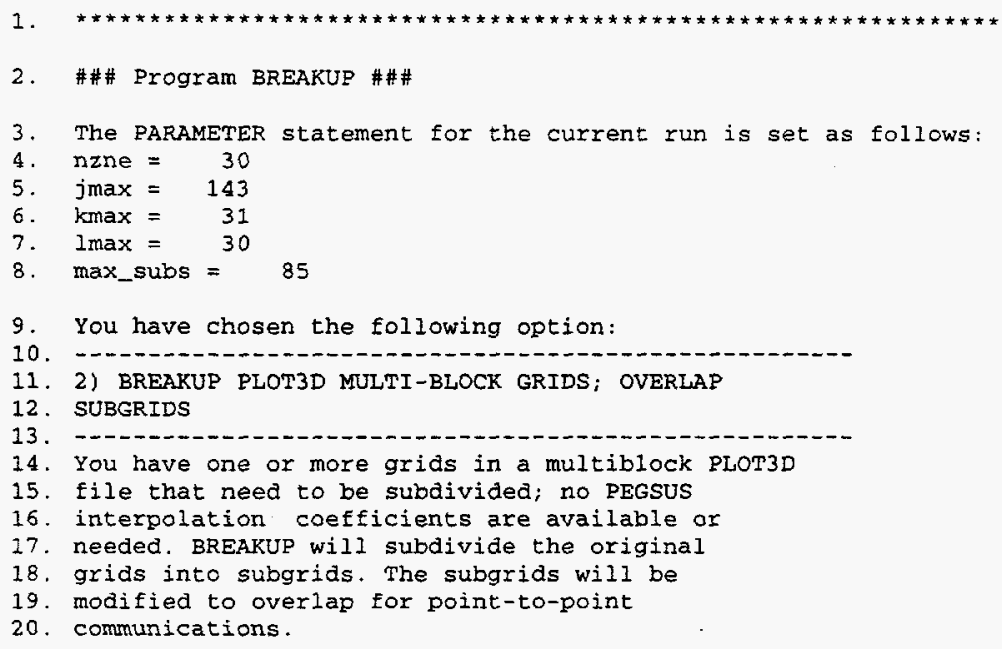

21. Read in grid. Grid is assumed to be in PLOT3D

22. format. BREAKUP can discern if file is

23. formatted or binary, and if grids have the

24. iblank array. Subgrids will be output to file

25. GRID_DIVIDED.G in formatted PLOT3D format.

26. input grid file must have one of the

27. following names:

28. X.FMT

29. X.DAT

30. XY.FMT

31. XY.DAT

32. XYZ.EMT

33. XYZ.DAT

34. GRID.FMT

35. GRID.DAT

36. G.FMT

37. G.DAT

38. Attempting to open file named: $X Y$.FMT

39. File opened successfully.

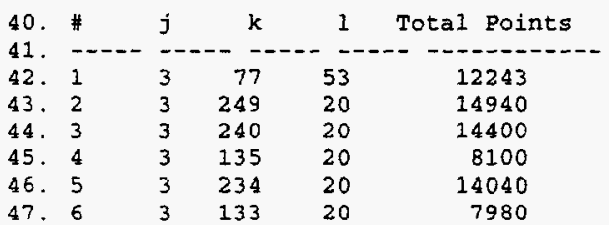

48. Zone 2 has the largest number of grid points:

49. $j \max =3$

50. $k \max =249$

51. $1 \max =20$

52. Total number of grid points for this zone: 14940

53. End of grid-file scan 


\begin{tabular}{|c|c|c|c|c|c|c|c|c|c|c|}
\hline 55. & $n z=$ & 1 & $j \max$ & $=$ & 3 & $k \max =$ & 77 & $1 \max$ & $=$ & 53 \\
\hline 56. & $n z=$ & 2 & $j \max$ & $=$ & 3 & $k \max =$ & 249 & $1 \max$ & $=$ & 20 \\
\hline 57. & $\mathrm{nz}=$ & 3 & $j \max$ & $=$ & 3 & $\mathrm{kmax}=$ & 240 & $I \max$ & $=$ & 20 \\
\hline 58. & $n z=$ & 4 & $j \max$ & $=$ & 3 & $k \max =$ & 135 & Imax & $=$ & 20 \\
\hline 59 & $\mathrm{nz}=$ & 5 & $j \max$ & $=$ & 3 & $k \max =$ & 234 & $1 \max$ & $=$ & 20 \\
\hline 60. & $n z=$ & 6 & $j \max$ & $=$ & 3 & $\mathrm{kmax}=$ & 133 & $1 \max$ & $=$ & 20 \\
\hline
\end{tabular}

61. Total number of grid points $=71703$

62.

63. Grid Jmax $\mathrm{Kmax} I \max$ Total

64. GRID0001.G $33^{7} \quad 53 \quad 12243$

65. GRIDO002.G $33249 \quad 20 \quad 14940$

66. GRID0003.G $3 \quad 240 \quad 20 \quad 14400$

67. GRID0004.G $3.135-20 \quad 8100$

68. GRIDO005.G $3 \quad 234 \quad 20 \quad 14040$

69. GRID0006.G $3133 \quad 20 \quad 798$

70. Total number of grid points $=71703$

71. Total number of original zones $=6$

72. Average number of grid points/grid $=11950.50$

73. Parallel processing parameters:

74. Number of nodes to be used:

16

75. Number of grid points in zone 1: 12243

76. Number of grid points in zone 2: 14940

77. Number of grid points in zone $3: \quad 14400$

78. Number of grid points in zone 4: 8100

79. Number of grid points in zone 5: 14040

80. Number of grid points in zone $6: \quad 7980$

81. Total number of grid points over all grids:

82. Average number of grid points per grid: 11950.5

83. Average number of grid points per processor: 4481.0
84. For zone 1 use 3 processors.
85. For zone 2 use 3 processors.
86. For zone 3 use 3 processors.
87. For zone 4 use 2 processors.
88. For zone 5 use 3 processors.
89. For zone 6 use 2 processors.

90. User-specified nodes to be used $=16$

91. Nodes calculated to be used $=16$

92. Calculate $j * k * . L$ values for subgrids for minimun

93. area-to-volume ratio.

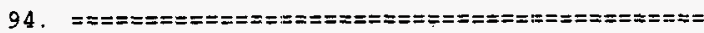

95. For mesh GRID0001.G:

96. List all possible factors for number of subgrids

97. for this zone.

$\begin{array}{rrrrr}98 . & \text { ITAG } & \text { J } & \text { K } & \text { L } \\ 99 . & -1 & -- & -- \\ 100 . & 1 & 1 & 1 & 3 \\ 101 . & 2 & 1 & 3 & 1 \\ 102 . & 3 & 3 & & 1\end{array}$

103. Finding min (surf/vol) for:

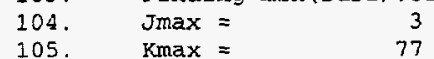

106. $\quad L \max =53$

107. itag surf/vol min(surf/vol)

$\begin{array}{llll}\text { 108. } & 1 & 0.805848 & 0.805848\end{array}$ 


$\begin{array}{llll}109 . & 2 & 0.782325 & 0.782325 \\ 110 . & 3 & 2.063710 & 0.782325\end{array}$

111

112 .

113.

114

115

116

117

118.

119.
120.

121.

122

123

124 .

125 .

126

127.

128

129

130

131.

132 .

133.

134.

135 .

136.

137.

138 .

239 .

140 .

141 .

142 .

143.

144.

145.

146

147.

148.

149.

150 .

151.

152 .

153

154.

155 .

156

157.

158.

159.

160 .

161.

162 .

163

164

165

166

167.

168.
169.

170

Final values for $\min (s u r f / v o l)$ for this zone are:

itag $=2$

$\min ($ surf $/$ vol $)=0.782325$

$\mathrm{N} / \mathrm{Jmax}=1 / 3=\Rightarrow$ approx. 3 pts/subgrid in $\mathrm{J}$ direction

$M / K \max =3 / 77=\Rightarrow$ approx. $26 \mathrm{pts} /$ subgrid in $\mathrm{K}$ direction

$\mathrm{P} / \mathrm{Lmax}=1 / 53==$ approx, $53 \mathrm{pts} /$ subgrid in L direction

Warning: Kmaxx/M not evenly divisible!

$\mathrm{K}$-remainder $=1$

BREAKUP will make adjustments in the size of the subgrids to compensate for the above remainders.

Adjustments will start at the boundaries and

progress inward. Hence, when remainders appear,

outer subgrids will have their dimensions

increased in the corresponding direction.

Final subgrid dimensions are:

\begin{tabular}{|c|c|c|c|c|c|c|c|}
\hline Grid \# & Jcube & Kcube & Lcube & $J \max$ & $\mathrm{Kmax}$ & Lmax & Total \\
\hline 1 & 1 & 1 & 1 & 3 & 27 & 53 & 4293 \\
\hline 2 & 1 & 2 & 1 & 3 & 26 & 53 & 4134 \\
\hline 3 & 1 & 3 & 1 & 3 & 26 & 53 & 4134 \\
\hline
\end{tabular}

Compare total number of grid points with original

in this zone:

Subtracted common faces in $\mathrm{K}$-direction.

original no. of points in this zone = 12243

Calculated no, of points in this zone $=12243$

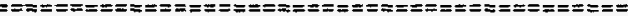

FOI mesh GRIDO002.G:

List all possible factors for number of subgrids

for this zone.

ITAG

ITAG

1

2

\begin{tabular}{|c|c|c|}
\hline $\mathcal{J}$ & K & L \\
\hline$\cdots$ & $\cdots$ & \\
\hline 1 & 1 & 3 \\
\hline 1 & 3 & 1 \\
\hline 3 & 1 & 1 \\
\hline
\end{tabular}

Finding $\min ($ surf/vol) for:

Jmax $=$

$\operatorname{Kmax}=$

$\mathrm{L} \max =$

249

itag

surf/vol

$\min$ (surf/vol)

I $\quad 0.974699 \quad 0.974699$

$2 \quad 0.790763 \quad 0.790763$

$3 \quad 2.108032 \quad 0.790763$

Final values for min(surf/vol) for this zone are:

itag $=$

$\min (\operatorname{surf} / \mathrm{vol})=0.790763$

$\mathrm{N} / \mathrm{J} \max =1 / 3 \Rightarrow$ approx. $\quad 3 \mathrm{pts} /$ subgrid in J direction

$\mathrm{M} / \mathrm{K} \max =3 / 249==>$ approx. 83 pts/subgrid in $\mathrm{K}$ direction

$P / L \max =1 / 20=\Rightarrow$ approx. 20 pts/subgrid in L direction

Warning: Kmaxx/M not evenly divisible!

$\mathrm{K}$-remainder $=2$

BREAKUP will make adjustments in the size of the

subgrids to compensate for the above remainders.

Adjustments will start at the boundaries and

progress inward. Hence, when remainders appear,

outer subgrids will have their dimensions

increased in the corresponding direction.

Final subgrid dimensions are:

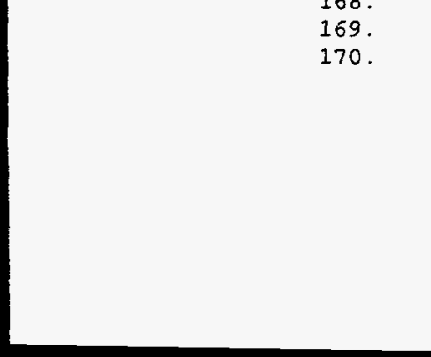

\begin{tabular}{|c|c|c|c|c|c|c|c|}
\hline Grid \# & Jcube & Kcube & Lcube & $J \max$ & $K \max$ & Lmax & Total \\
\hline 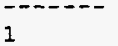 & $\cdots--$ & $\cdots--$ & $---\infty$ & $3^{---}$ & $-\cdots$ & $20^{---}$ & 5040 \\
\hline
\end{tabular}




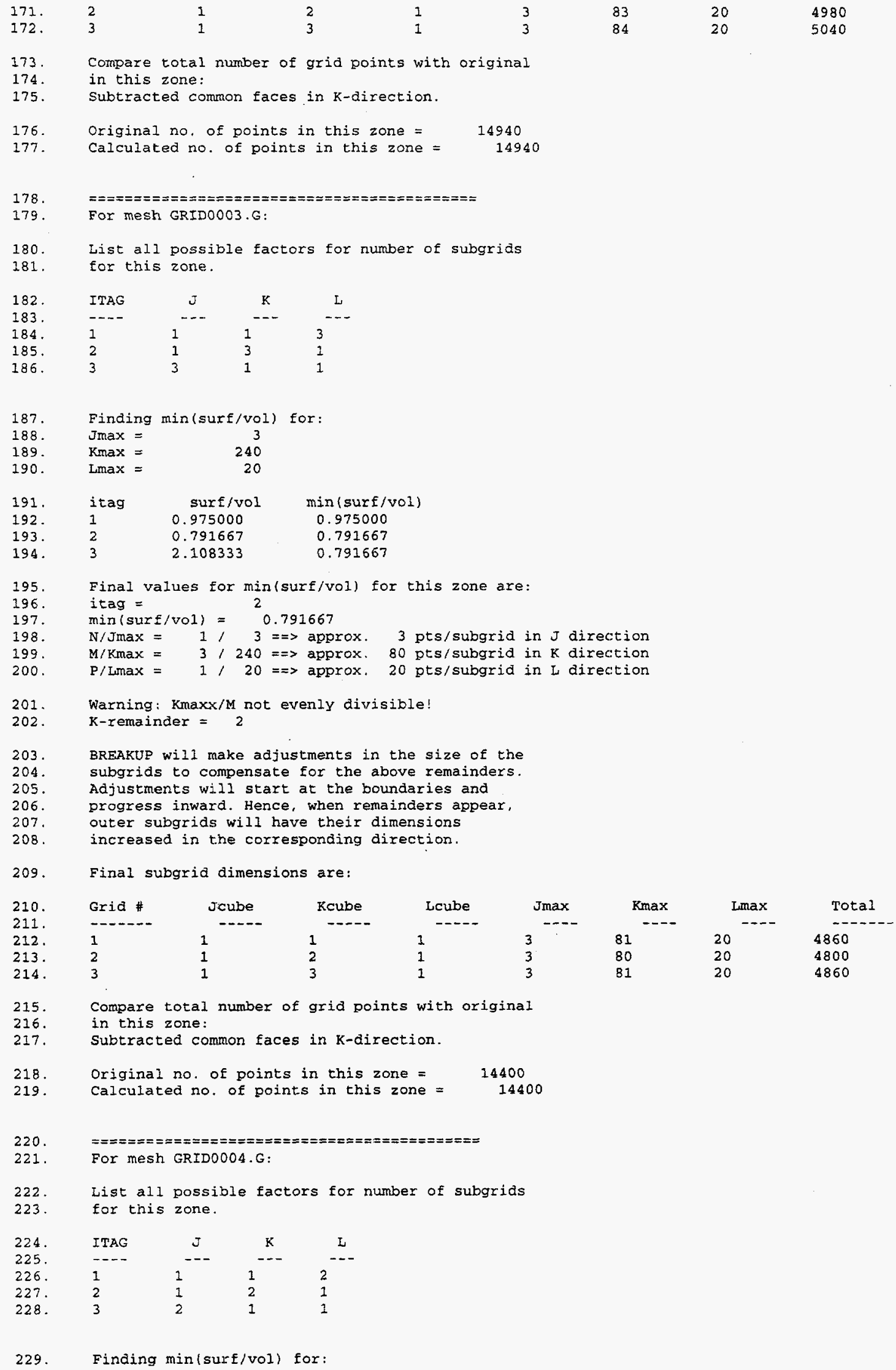

List all possible factors for number of subgrids

$\begin{array}{llll}\text { ITAG } & \text { J } & \text { K } & \text { L } \\ 1 & 1 & 1 & 2 \\ 2 & 1 & 2 & 1 \\ 3 & 2 & 1 & 1\end{array}$




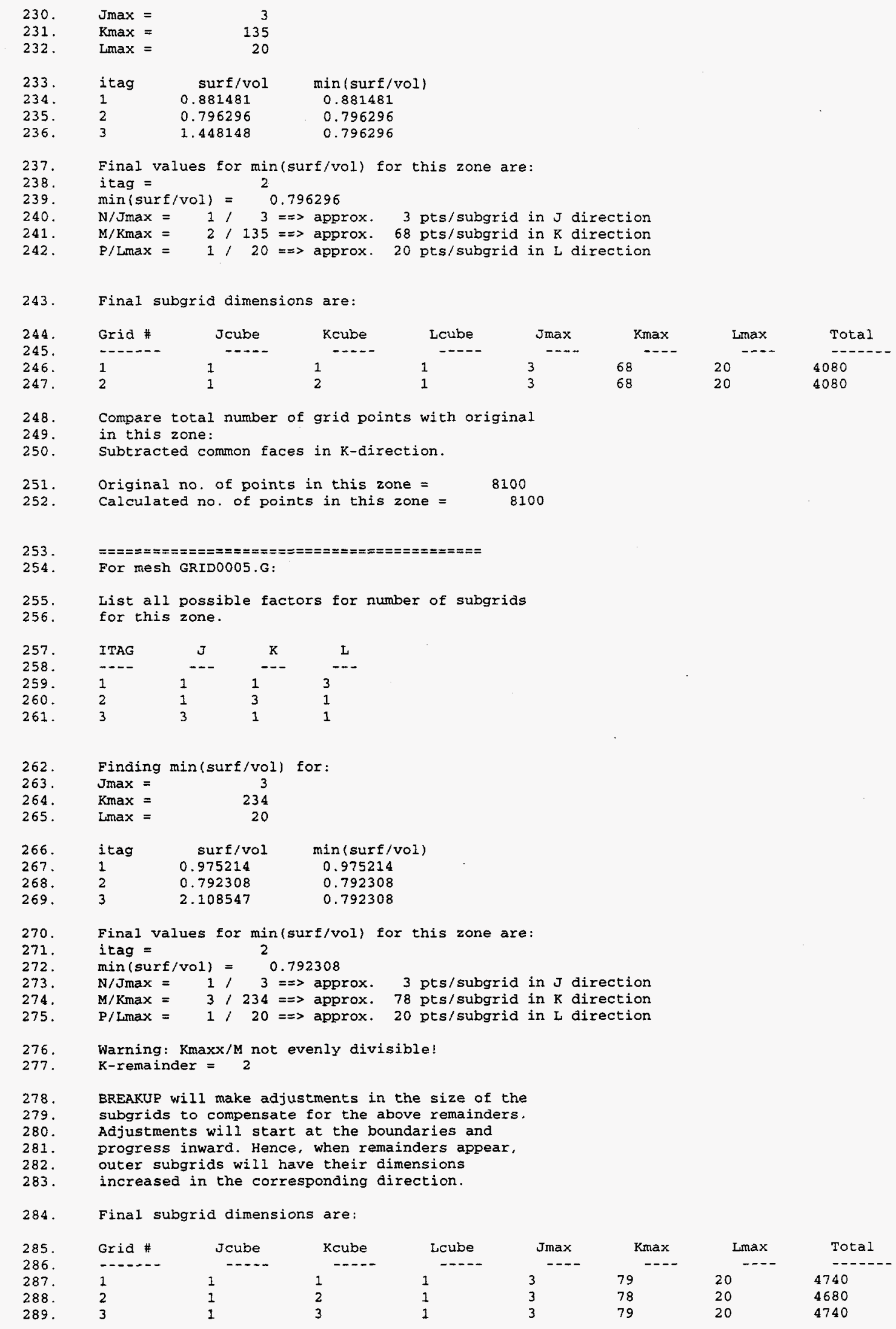

290. Compare total number of grid points with original 


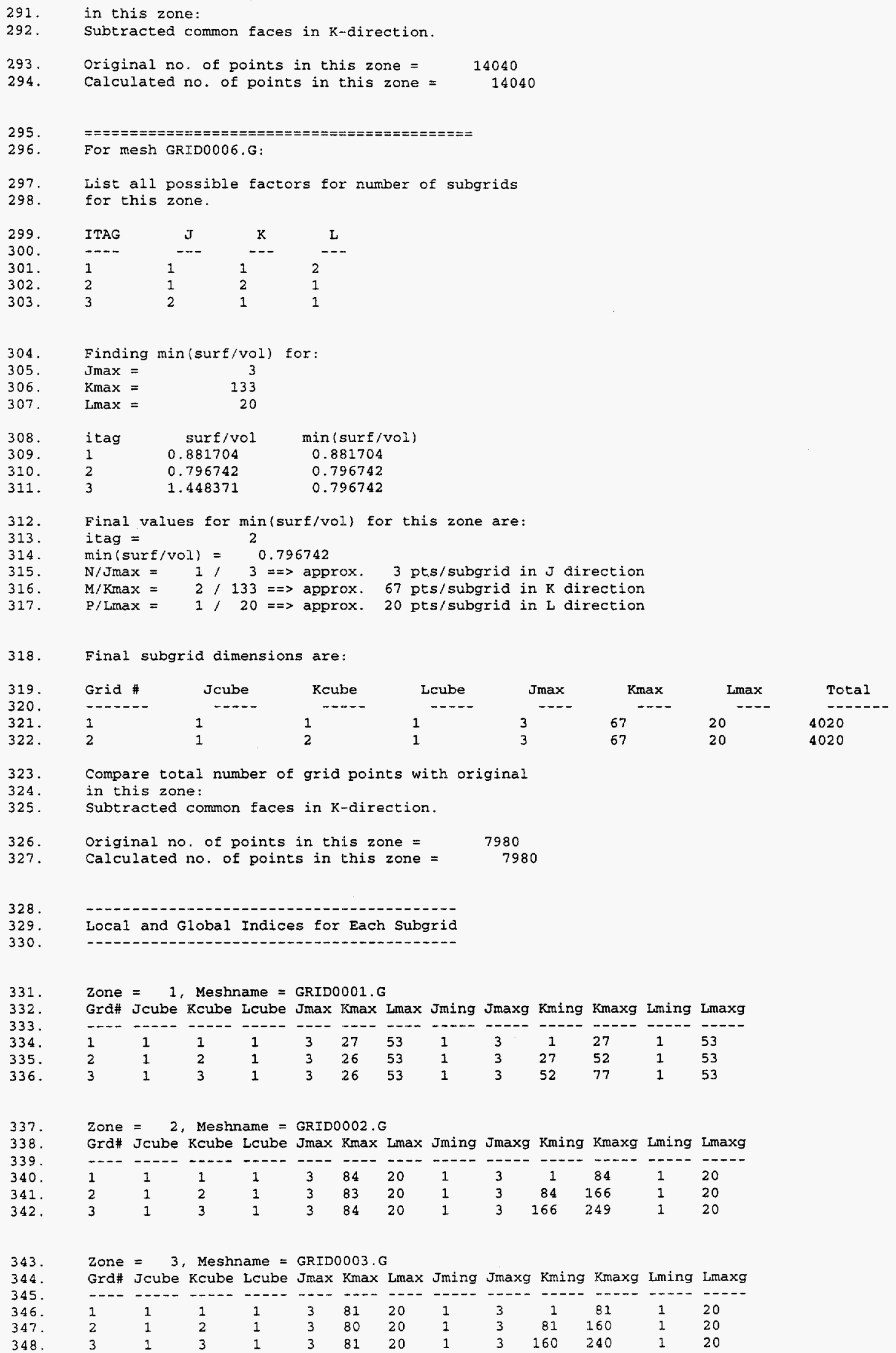


362 .

363.

364.

365.

366.

367 .

368 .

369 .

370.

371.

372.

373.

374.

375.

377.

378.

379.

380 .

381.

382 .

383.

384 .

385 .

386 .

387 .

388.

389.

390.

391.

392.

393.

394.

395.

396.

397.

398.

400 .

401.

402 .

403.

404.

405.
406.

407.

408 .

409 .

410 .

411.

Zone $=4$, Meshname $=$ GRID0004.G

Grd\# Jcube Kcube Lcube Jmax Kmax Lmax Jming Jmaxg Kming Kmaxg Lming Lmaxg

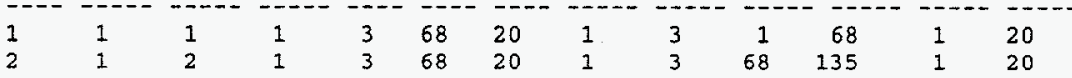

Zone $=5$, Meshname $=$ GRID0005.G

Grd\# Jcube Kcube Lcube Jmax Kmax Lmax Jming Jmaxg Kming Kmaxg Lming Imaxg

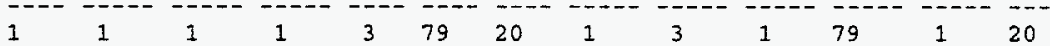

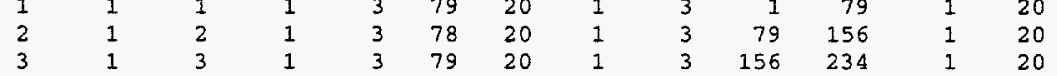

Zone $=6$. Meshname $=$ GRID0006.G

Grd\# Jcube Kcube Lcube Jmax Kmax Lmax Jming Jmaxg Kming Kmaxg Lming Lmaxg

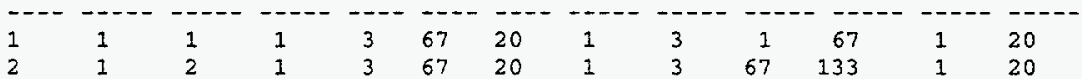

Breaking up grids into subgrids WITH NO overlap:

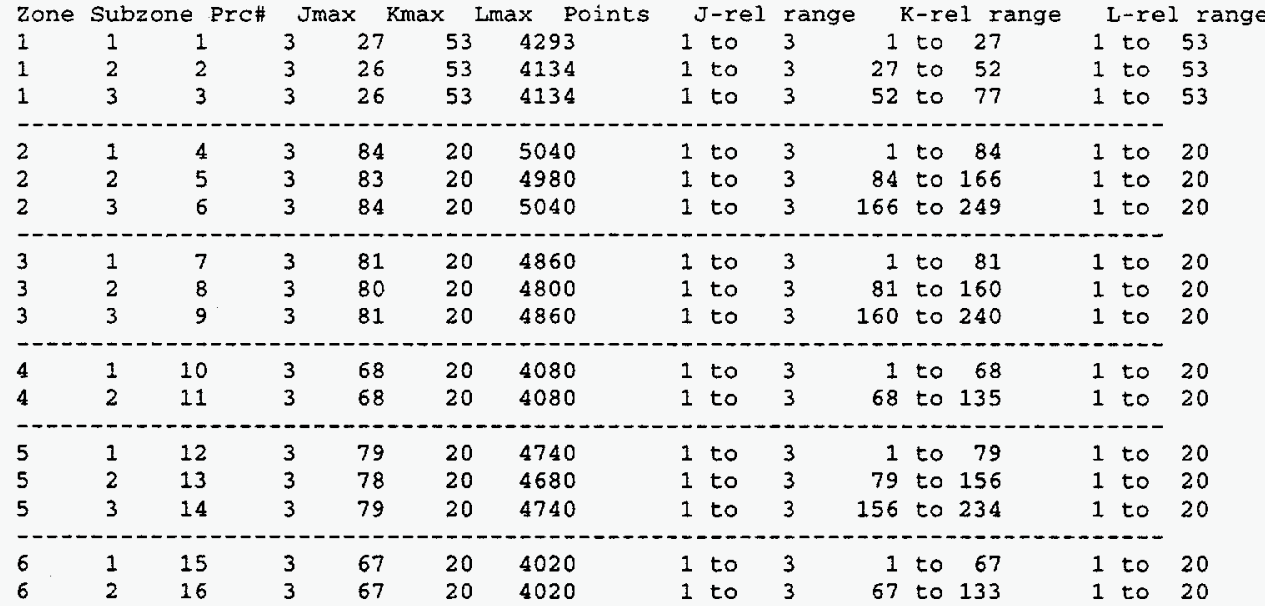

Breaking up grids into subgrids WITH overlap:

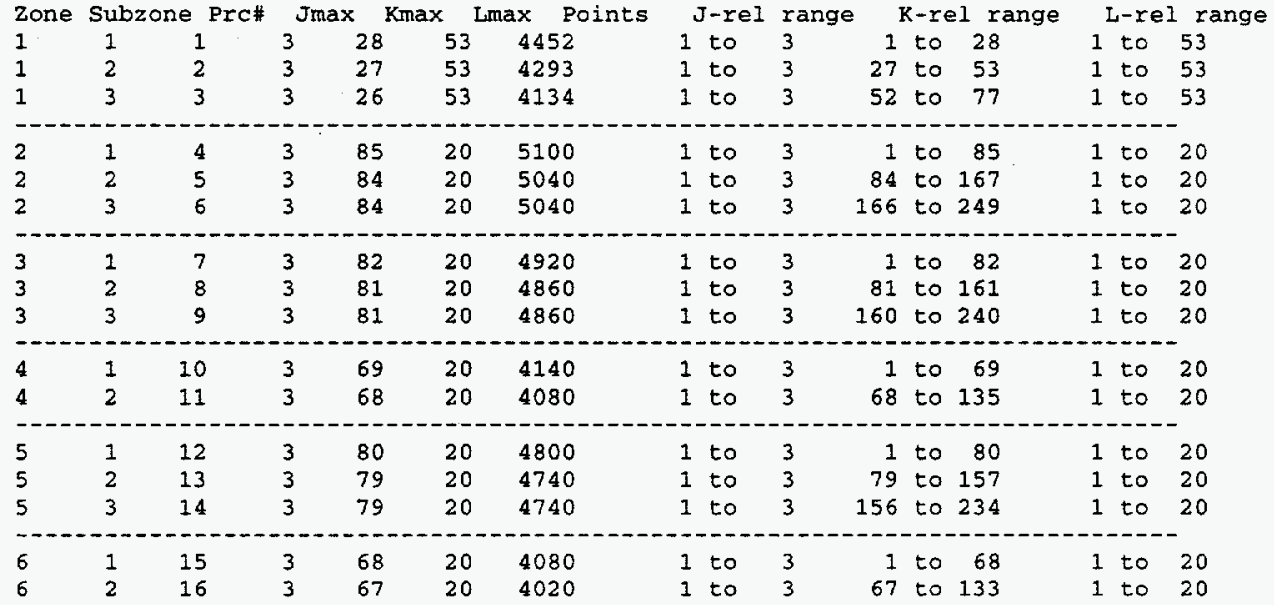

Writing subgrids to file.

Choose PLOT3D-output-format of file for subgrids: 
413. formatted
414. unformatted
415. Option number 1 chosen.

416. Choose whether you want the IBLANK array attached:

417. attach iblank array to subgrids

418. do NOT attach iblank array to subgrids

419. Option number 1 chosen.

420. Output file GRID_DIVIDED.G opened as formatted.

421. Constructing subgrids.

422 .

423.

424 .

Reading original grid file, zone \#

This grid contains IBLANK data which will be read.

426 .

427 .

428 .

429

430 .

431.

432 .

433

434.

This grid contains IBLANk data which wild be read.

Constructing subgrid \# 1 of 3 subgrids in zone 1

Total count: subgrid \# 1 of 16 subgrids

Grid cells overlap 1 cell widths on grid boundaries. IBLANK data available and will be written to

this subgrid file.

$\mathrm{nz}=1$ jcube $=1$ kcube $=1$ lcube $=1$

jp3d_min $=1$ jp3d_max $=3$

$\mathrm{kp} 3 \mathrm{~d}_{\text {_min }}=1 \mathrm{kp} 3 \mathrm{~d}$ _max $=28$

$1 p 3 d \_$min $=1$ lp3d_max $=53$

Constructing subgrid \# 2 of 3 subgrids in zone

Total count: subgrid \# 2 of 16 subgrids

Grid cells overlap 1 cell widths on grid boundaries.

IBLANK data available and will be written to

this subgrid file.

$\mathrm{nz}=1$ jcube $=1$ kcube $=2$ lcube $=1$

jp3d min $=1$ jp3d $\max =3$

kp3d_min $=27 \mathrm{kp3d \_ max}=53$

1p3d_min $=1$ lp3d_max $=53$

Constructing subgria \# 3 of 3 subgrids in zone 1

Total count: subgrid \# 3 of 16 subgrids

Grid cells overlap 1 cell widths on grid boundaries.

IBLANK data available and will be written to

this subgrid file.

$\mathrm{nz}=1$ jcube $=1$ kcube $=3$ Icube $=1$

jp3d_min $=1$ jp3d_max $=3$

kp3d_min $=52$ kp3d_max $=77$

lp3d_min $=1$ lp3d_max $=53$

453.
454.
455.

Reading original grid file, zone \# 2

This grid contains IBLANK data which will be read.

456 .

457 .

458 .

459 .

460 .

461 .

462 .

463 .

464 .

465 .

Constructing subgrid \# 1 of 3 subgrids in zone Total count: subgrid \# 4 of 16 subgrids

Grid celis overlap 1 cell widths on grid boundaries. IBLANK data available and will be written to this subgrid file.

$\mathrm{nz}=2$ jcube $=1$ kcube $=1$ lcube $=1$

jp3d_min $=\quad 1$ jp3d_max $=3$

kp3d_min $=1$ kp3d_max $=85$

$1 p 3 d \min =1$ lp3d_max $=20$

Constructing subgrid \# 2 of 3 subgrids in zone

Total count: subgrid \# 5 of 16 subgrids

Grid cells overlap 1 cell widths on grid boundaries.

IBLANK data available and will be written to

this subgrid file.

$\mathrm{nz}=2$ jcube $=$

1 kcube $=$

2 lcube $=1$

jp3d_min $=$ I jp3d_max $=3$

$\mathrm{kp} 3 \mathrm{~d} \min =84 \mathrm{kp} 3 \mathrm{~d} \max =167$

lp3d_min $=\quad 1$ lp3d_max $=20$ 
475. Constructing subgrid \# 3 of 3 subgrids in zone 2

476. Total count: subgrid \# 6 of 16 subgrids

477. Grid cells overlap 1 cell widths on grid boundaries.

478. IBLANK data available and will be written to

479. this subgrid file.

480. $\mathrm{nz}=2$ jcube $=1$ kcube $=3$ lcube $=1$

481. jp3a_min $=1$ jp3d_max $=3$

482. $\quad k p 3 d_{\text {_min }}=166 \mathrm{kp} 3 \mathrm{~d}_{\max }=249$

483. 1p3d_min $=1$ 1p3d_max $=20$
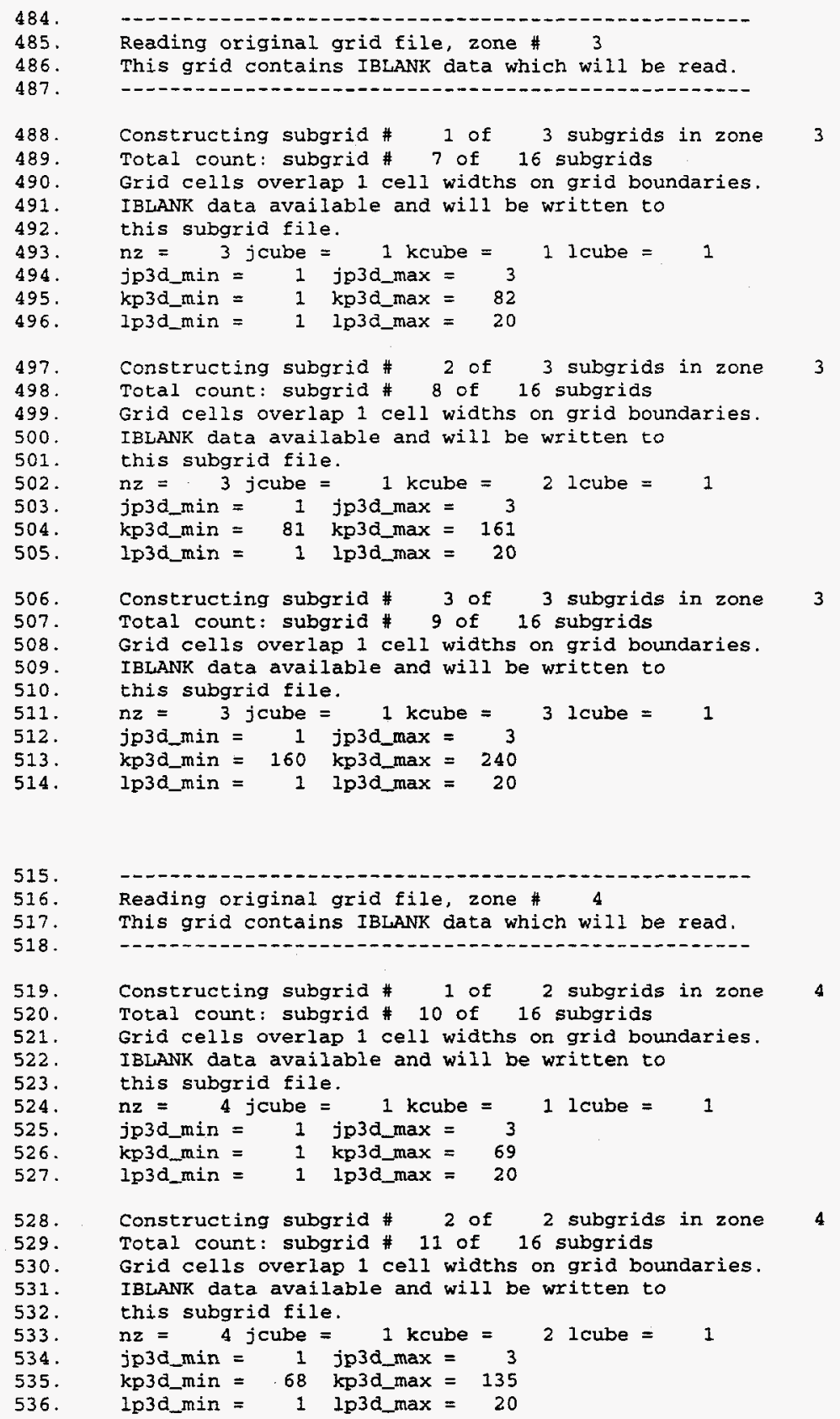

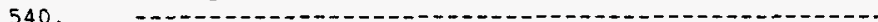


541. Constructing subgrid \# 1 of 3 subgrids in zone

542. Total count: subgrid \# 12 of 16 subgrids

543. Grid cells overlap 1 cell widths on grid boundaries.

544. IBLANK data available and will be written to

545 ." this subgrid file.

546. $\mathrm{nz}=5$ jeube $=1$ kcube $=1$ icube $=1$

547. jp3d_min $=1$ jp3d_max $=3$

548. $\quad$ kp3d_min $=1$ kp3d_max $=80$

549. 1p3d_min $=1$ lp3d_max $=20$

550. Constructing subgrid \# 2 of 3 subgrids in zone

551. Total count: subgrid \# 13 of 16 subgrids

552. Grid cells overlap 1 cell widths on grid boundaries.

553. IBLANK data available and will be written to

554. this subgrid file.

555. $\mathrm{nz}=5$ jeube $=1$ kcube $=2$ lcube $=1$

556. jp3d_min $=1$ jp3d_max $=3$

557. kp3d_min $=79$ kp3d_max $=157$

558. Ip3̄d_min $=1$ lp3d_max $=20$

559. Constructing subgrid \# 3 of 3 subgrids in zone

560. - Total count: subgrid \# 14 of 16 subgrids

561. Grid cells overlap 1 cell widths on grid boundaries.

562. IBLANK data available and will be written to

563 . this subgrid file.

564. $\mathrm{nz}=5$ jcube $=1$ kcube $=3$ lcube $=1$

565. jp3d_min $=1$ jp3d_max $=3$

566. kp3d_min $=156$ kp3d_max $=234$

$567 \quad$ lp $3 \mathrm{~d} \min =1$ lp3d_max $=20$

Reading original grid file, zone \# 6

This grid contains IBLANK data which will be read.

571

Constructing subgrid

1 of

2 subgrids in zone

573.

574

575 .

576

577 .

578

579

580 .

581.

582 .

582.

583.

585

586 .

587 .

588

589

Total count: subgrid \# 15 of 16 subgrids

Grid cells overlap 1 cell widths on grid boundaries.

IBLANK data available and will be written to

this subgrid file.

$\mathrm{nz}=6$ jcube $=$

jp3d_min $=1$ jp3d max $=$

$\mathrm{kp} 3 \mathrm{~d} \min =1 \mathrm{kp} 3 \mathrm{~d}_{\max }=68$

Ip3d_min $=1$ lp3d_max $=20$

Constructing subgrid \# 2 of 2 subgrids in zone

Total count: subgrid \# 16 of 16 subgrias

Grid cells overlap 1 cell widths on grid boundaries.

IBLANK data available and will be written to

this subgrid file.

$\mathrm{nz}=6$ jcube $=1$ kcube $=2$ lcube $=1$

jp3d_min $=1$ jp3d_max $=3$

$\mathrm{kp} 3 \mathrm{~d}_{\min }=67 \mathrm{kp} 3 \mathrm{~d} \_\max =133$

$1 p 3 \mathrm{~d} \min =1$ 1p3d_max $=20$

590.

591.

591.

593.

Screen output directed to fj.le: BREAKUP.OUT

Subgrids output to file: GRID_DIVIDED.G

in PLOT3D Eormat.

595. $\quad>$ Finished $\ll$

596.

Program BREAKUP finished. 
Micro Craft

Attn: J. A. Benek (1)

N. Suhs (4)

207 Big Springs Avenue

P.O. Box 370

Tullahoma, TN 37388-0370

1

1 M. Remotigue

NSF/ERC at MSU

MS 962

ERC 203

P.O. Box 9627

Mississippi State, MS 39762

1 Dr. Ray Gomez

Advanced Programs Office

Mail Code EG3

NASA Johnson Space Center

Houston, TX 77058

1

Prof. David Whitfield

Engineering Research Center

Mississippi State University

P.O. Box 9627

Mississippi State, MS 39762

3

Dr. Bob Meakin

NASA Ames Research Center

MS 258-1

Moffett Field, CA 94035-1000

$5 \quad$ Lockheed Martin Skunkworks

Attn: G. Shrewsbury (4)

J. Vadyak (1)

Computational Aerodynamics

1011 Lockheed Way

Palmdale, CA 93599-2523

1

Mr. D. Howlett
Dr. Dave Bader

Battelle Washington Operations

901 D Street SW, Suite 900

Washington, DC 20024-2115

1

Dr. Pieter Buning

Configuration Aerodynamics Branch

MS 499

NASA Langley Research Center

Hampton, VA 23681-0001

1 Dr. F. C. Dougherty

Supercomputer Computations Research Institute

Florida State University

Tallahassee, FL 32306-4052

1

Dr. Stuart Rogers

Mail Stop 227-2

NASA Ames Research Center

Moffett Field, CA 94035-1000

3 Charlie Nietubicz, Director

Major Shared Resource Center

US Army Research Laboratory

Aberdeen Proving Ground, MD 21005-5067

1 Dennis Jespersen

MS T27B-1

NASA Ames Research Center

Moffett Field, CA 94035-1000

2

CFD Research Corporation

Attn: Sami D. Habchi

215 Wynn Drive

Huntsville, AL 35805

2
Dr. Michelle Hribar

Dr. Jerry C. Yan

MS T27A-2

NASA Ames Research Center

Moffett Field, CA 94035-1000

Engineering Chief

Aerodynamics \& Computational Fluid Dynamics

Lockheed Martin Tactical Aircraft Systems

PO Box 748

Mail Zone 9333

Ft. Worth, TX 76101 
INTERNAL DISTRIBUTION:

\begin{tabular}{|c|c|c|}
\hline MS & 0318 & G. S. Davison, 9215 \\
\hline & 0321 & W. J. Camp, 9200 \\
\hline & 0439 & D. R. Martinez, 9234 \\
\hline & 0441 & P. Knupp, 9226 \\
\hline & 0441 & R. W. Leland, 9226 \\
\hline & 0819 & J. Peery, 9231 \\
\hline & 0820 & P. Yarrington, 9232 \\
\hline & 0825 & B. Hassan, 9115 \\
\hline & 0825 & J. Payne, 9115 \\
\hline & 0825 & F. Blottner, 9115 \\
\hline & 0825 & W. Rutledge, 9115 \\
\hline & 1109 & A. L. Hale, 9224 \\
\hline & 1110 & D. Greenburg, 9223 \\
\hline & 1110 & D. E. Womble, 9222 \\
\hline & 1111 & S. S. Dosanjh, 9221 \\
\hline & 1111 & D. W. Barnette, 9221 \\
\hline & 1111 & G. Heffelfinger, 9225 \\
\hline & 1166 & J. D. Kotulski, 9352 \\
\hline & 1166 & D. J. Riley, 9352 \\
\hline & 1166 & D. C. Turner, 9352 \\
\hline & 0161 & Patent \& Licensing Office, 11500 \\
\hline & 9018 & Central Technical Files, $8940-2$ \\
\hline & 0899 & Technical Library, 4619 \\
\hline & 0619 & $\begin{array}{l}\text { Review \& Approval Desk, } 12690 \\
\text { For DOE/OSTI }\end{array}$ \\
\hline
\end{tabular}

